\title{
Pore-scale study of counter-current imbibition in strongly water-wet fractured porous media using lattice Boltzmann method
}

\author{
Qingqing Gu(顾青青), ${ }^{1}$ Lianhua Zhu(朱炼华), ${ }^{1}$ Yonghao Zhang(张勇豪), ${ }^{1}$ and Haihu Liu(刘海湖) ${ }^{2}$, a) \\ 1) James Weir Fluids Laboratory, Department of Mechanical and Aerospace Engineering, University of Strathclyde, \\ Glasgow G1 1XJ, UK \\ ${ }^{2)}$ School of Energy and Power Engineering, Xi'an Jiaotong University, 28 West Xianning Road, Xi'an 710049, \\ China
}

(Dated: 9 July 2019)

Oil recovery from naturally fractured reservoirs with low permeability rock remains a challenge. To provide a better understanding of spontaneous imbibition, a key oil recovery mechanism in the fractured reservoir rocks, a pore-scale computational study of the water imbibition into an artificially generated dual-permeability porous matrix with a fracture attached on top is conducted using a recently improved lattice Boltzmann color-gradient model. Several factors affecting the dynamic counter-current imbibition processes and the resulting oil recovery have been analyzed, including the water injection velocity, the geometry configuration of the dual permeability zones, interfacial tension, viscosity ratio of water to oil phases, and fracture spacing if there are multiple fractures. Depending on the water injection velocity and interfacial tension, three different imbibition regimes have been identified: the squeezing regime, the jetting regime and the dripping regime, each with a distinctively different expelled oil morphology in the fracture. The geometry configuration of the high and low permeability zones affects the amount of oil that can be recovered by the counter-current imbibition in a fracture-matrix system through transition of the different regimes. In the squeezing regime, which occurs at low water injection velocity, the build-up squeezing pressure upstream in the fracture enables more water to imbibe into the permeability zone closer to the fracture inlet thus increasing the oil recovery factor. A larger interfacial tension or a lower water-to-oil viscosity ratio is favorable for enhancing oil recovery and new insights into the effect of viscosity ratio are provided. Introducing an extra parallel fracture can effectively increase the oil recovery factor and there is an optimal fracture spacing between the two adjacent horizontal fractures to maximize the oil recovery. These findings can aid the optimal design of water-injecting oil extraction in fractured rocks in reservoirs like oil shale.

\section{INTRODUCTION}

Fractured petroleum reservoirs typically represent over $20 \%$ of the world's oil reserves ${ }^{1}$. Such reservoirs are poorly understood and oil recovery from fractured reservoirs is typically lower than that from conventional reservoirs. For conventional reservoirs, water flooding is one of the primary techniques to enhance oil recovery (EOR), in which water is injected to displace oil from the porous matrix under the pressure difference. However, for the low permeable fractured reservoirs, the water-flooding displacement is often not productive because the limited connectivity among the pores cannot guide effectively the flow of the expelled oil along the pressure gradient ${ }^{2}$.

Yet still, in many naturally fractured reservoirs, the spontaneous imbibition (also known as capillary imbibition) provides a special mechanism for oil recovery from the matrix which does not rely on the imposed pressure gradient. In the spontaneous imbibition, the wetting fluid is imbibed into the porous rock matrix due to the capillary pressure without any external driving force. The oil in the rock matrix is expelled and flows into the fractures due to the spontaneous imbibition of water into the matrix from the fractures. The spontaneous imbibition can be either co-current and counter-current, depending on whether the water and oil transport in the same (co-current) or opposite (counter-current) directions ${ }^{3}$, as il-

\footnotetext{
${ }^{a)}$ Electronic mail: haihu.liu@ mail.xjtu.edu.cn
}

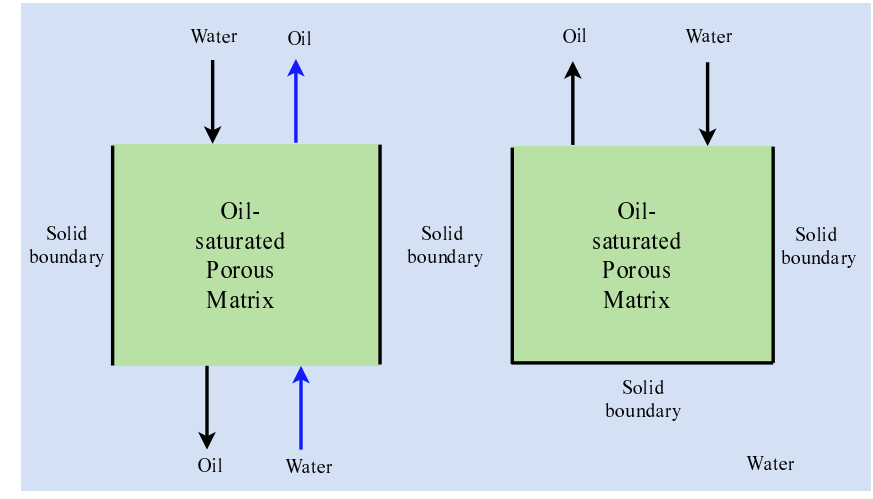

(a)

FIG. 1. Schematic of the (a) co-current imbibition: the expelled oil and the imbibing water (see arrows in the same color) flow in the same direction, and (b) counter-current imbibition: the expelled oil and the imbibing water (black arrows) flow in the opposite directions.

lustrated in Fig. 1. In this work, we focus on the countercurrent imbibition [Fig. 1(b)], where the wetting phase and non-wetting phase flow in/out of the matrix at the same side, and enclosed (by wall) matrix is necessary for such flow to occur. The counter-current imbibition occurs more often although it is less effective in terms of oil recovery. The spontaneous imbibition is known to be affected by many factors including the wettability ${ }^{4}$, the porosity, permeability and heterogeneity of the matrix ${ }^{5-11}$, the viscosity ratio of the wetting 
to non-wetting fluids ${ }^{12,13}$ and their interfacial tension ${ }^{14,15}$, as well as initial saturation and boundary conditions ${ }^{10,14,16}$, etc.

The spontaneous imbibition in conventional porous media has been extensively studied ${ }^{17-20}$ due to its wide existence in various disciplines such as oil recovery, polymer composite manufacturing, soil science and subsurface hydrology. For the fractured porous medium, however, it has not been fully understood and starts to attract research attention. RangelGerman and Kovscek ${ }^{21}$ experimentally investigated the underlying flow physics of the counter-current imbibition in various fracture apertures using an X-ray computerized tomography (CT) scanner. By changing the water injection rates through the fractures, they have identified two spontaneous imbibition regimes, i.e., the "filling fracture" and the "instantly filled fracture" regimes which correspond to significantly different imbibition behaviors. Later, they further verified such a finding by micromodel experiments with the aid of pore-scale imaging techniques. Jafari et al. ${ }^{22}$ studied the effect of fracture network topology, pore sizes distribution and structure of matrix and injection rate on the spontaneous imbibition using a glass micromodel.

In addition to experimental studies, pore-scale numerical simulations have also been exploited to understand spontaneous imbibition. Pore-scale simulations allow easy access to a wide range of parameters and the imbibition process can be readily visualized so that its underlying mechanism can be analyzed accordingly in a more controllable manner. Moreover, numerical simulations are superior to theoretical analysis when it comes to geometrically complex problems with nonlinearity ${ }^{9}$. Rokhforouz and Amiri ${ }^{4}$ used the phase-field method to study the effect of wettability, interfacial tension, and viscosity ratio during counter-current imbibition process. Jafari et $\mathrm{al}^{9}$ also used the phase-field method to investigate the effects of water injection velocity, fracture aperture, and grain shape during counter-current spontaneous imbibition. In these pore-scale simulations, the solid grains in the matrix are generally idealized as simple circular ${ }^{4,9}$ or square cylinders ${ }^{23}$. However, the pore structures in natural rock matrix are extremely complex and irregular. In this work, we conduct the pore-scale study of the counter-current imbibition in a matrixfracture system using a recently improved lattice Boltzmann (LB) color-gradient model ${ }^{24}$. Among various multiphase LB models (see the reviews by Huang et al. ${ }^{25}$ and Liu et al. ${ }^{26}$ ), the color-gradient model has its own advantages such as high numerical accuracy, strict mass conservation for each fluid and numerical stability for a broad range of viscosity ratios ${ }^{24}$. Especially, it produces relatively thin interface and is able to control the interfacial diffusion and adjust the interfacial tension and viscosity independently to facilitate the numerical investigation $^{27}$. Therefore, the color-gradient model has been extensively used for modeling immiscible two phase flow in porous media, e.g. Tolke et al. ${ }^{28}$, Huang et al. ${ }^{29}$, Chen et al. ${ }^{30}$, Gu et al. ${ }^{27}$ and $\mathrm{Xu}$ et al. ${ }^{31}$.

Here, the matrix is constructed using a Voronoi tessellation technique where the pore structure is simplified as randomly connected throats. The throat widths are specified according to the log-normal distribution, which was found to be a good geometric approximation to natural porous media in statis- tics $^{32}$. Also, the matrix is constructed with two different permeability zones along the fracture as commonly encountered in multiplayer geological formations ${ }^{33}$. The effect of water injection velocity, geometry configuration of the dual permeability zones, interfacial tension, viscosity ratio, and fracture spacing (only when multiple fractures are present) is systematically studied, and the sensitivity analysis is performed to find the optimal parameters that maximise the oil recovery.

\section{NUMERICAL METHOD}

The LB color-gradient model is employed to solve the counter-current spontaneous imbibition processes in this work. It is based on the work of Halliday et al. ${ }^{34-36}$ and incorporates the improvements given by Xu et al. ${ }^{24}$ and Yu et al. ${ }^{37}$. The indicator or color function $\rho^{N}$ is used to distinguish one fluid from the other, and it is defined as

$$
\rho^{N}(\boldsymbol{x}, t)=\frac{\rho^{R}(\boldsymbol{x}, t)-\rho^{B}(\boldsymbol{x}, t)}{\rho^{R}(\boldsymbol{x}, t)+\rho^{B}(\boldsymbol{x}, t)},
$$

where $\rho^{R}$ and $\rho^{B}$ are the densities of the red and blue fluids, respectively; $\boldsymbol{x}$ is the position and $t$ is the time. With this definition, $\rho^{N}=1$ and -1 represent the red fluid (oil) and the blue fluid (water), respectively, and $-1<\rho^{N}<1$ represents the diffuse interface where red and blue fluids coexist. For the two-dimensional 9-velocity (D2Q9) model used in this work, the lattice velocity vector is given by

$$
c_{i}= \begin{cases}(0,0), & i=0 \\ ( \pm 1,0) c,(0, \pm 1) c, & i=1,2,3,4 \\ ( \pm 1, \pm 1) c, & i=5,6,7,8\end{cases}
$$

where $c=\delta_{x} / \delta_{t}$ is the lattice speed with $\delta_{x}$ being the lattice length and $\delta_{t}$ being the time step. To allow for unequal viscosities of the two fluids and at the same time to ensure the continuity of viscosity flux across the interface ${ }^{38}$, the following harmonic mean is employed to determine the viscosity of the fluid mixture:

$$
\frac{1}{\eta}=\frac{1+\rho^{N}}{2 \eta^{R}}+\frac{1-\rho^{N}}{2 \eta^{B}}
$$

where $\eta^{k}(k=R$ or $B)$ is the dynamic viscosity of fluid $k$, which is related to the dimensionless relaxation time $\tau^{k}$ by $\eta^{k}=c_{s}^{2} \rho^{k}\left(\tau^{k}-0.5\right) \delta_{t}$. Here $c_{s}=c / \sqrt{3}$ is the lattice sound speed. The distribution functions $f_{i}^{R}$ and $f_{i}^{B}$ are introduced to represent the red and blue fluids. The total distribution function is defined as $f_{i}=f_{i}^{R}+f_{i}^{B}$. Each colored distribution function undergoes the collision and streaming steps as follows:

$$
f_{i}^{k}\left(\boldsymbol{x}+\boldsymbol{c}_{i} \boldsymbol{\delta}_{t}, t+\boldsymbol{\delta}_{t}\right)=f_{i}^{k}(\boldsymbol{x}, t)+\Omega_{i}^{k}\left(f_{i}^{k}(\boldsymbol{x}, t)\right),
$$

where $i$ denotes the discrete velocity directions, and $\Omega_{i}^{k}$ is the collision operator. The collision operator consists of three parts, and it is written as $\Omega_{i}^{k}=\left(\Omega_{i}^{k}\right)^{(3)}\left(\left(\Omega_{i}^{k}\right)^{(1)}+\left(\Omega_{i}^{k}\right)^{(2)}\right)$, where $\left(\Omega_{i}^{k}\right)^{(1)}$ is the single-phase collision operator, $\left(\Omega_{i}^{k}\right)^{(2)}$ 
is the perturbation operator which generates an interfacial tension, and $\left(\Omega_{i}^{k}\right)^{(3)}$ is the recoloring operator.

Compared to the Bhatnagar-Gross-Krook (BGK) approximation, the multiple relaxation time (MRT) model ${ }^{39-41}$ offers significant advantages such as reduced spurious velocities and enhanced numerical stability at low viscosities ${ }^{42}$. As a special MRT model with only two relaxation rates, the two-relaxation-time (TRT) model can produce viscosityindependent wall locations even with a relatively coarse grid resolution and is thus often used in the simulation of flows through porous media ${ }^{43-45}$. Note that the single-phase collision operator and the perturbation operator can be implemented by means of total distribution function. With the TRT model, the single-phase collision operator is expressed as

$$
\left(\Omega_{i}\right)^{(1)}=-\left(\mathbf{M}^{-1} \mathbf{S M}\right)_{i j}\left(f_{j}-f_{j}^{e q}\right),
$$

where the equilibrium distribution function $f_{i}^{e q}(\boldsymbol{x}, t)$ is given by:

$$
f_{i}^{e q}(\rho, \boldsymbol{u})=\rho W_{i}\left[1+\frac{\boldsymbol{c}_{i} \cdot \boldsymbol{u}}{c_{s}^{2}}+\frac{\left(\boldsymbol{c}_{i} \cdot \boldsymbol{u}\right)^{2}}{2 c_{s}^{4}}-\frac{\boldsymbol{u}^{2}}{2 c_{s}^{2}}\right] .
$$

Herein, $\rho=\rho^{R}+\rho^{B}$ is the total density, $\boldsymbol{u}$ is the local fluid velocity and the weight coefficients are $W_{0}=4 / 9, W_{1-4}=1 / 9$ and $W_{5-8}=1 / 36$.

The transformation matrix $\mathbf{M}$ is used to transform the distributions $f_{i}$ from the discrete velocity space into the moment space, and it is given by ${ }^{46}$

$$
\mathbf{M}=\left(\begin{array}{ccccccccc}
1 & 1 & 1 & 1 & 1 & 1 & 1 & 1 & 1 \\
-4 & -1 & -1 & -1 & -1 & 2 & 2 & 2 & 2 \\
4 & -2 & -2 & -2 & -2 & 1 & 1 & 1 & 1 \\
0 & 1 & 0 & -1 & 0 & 1 & -1 & -1 & 1 \\
0 & -2 & 0 & 2 & 0 & 1 & -1 & -1 & 1 \\
0 & 0 & 1 & 0 & -1 & 1 & 1 & -1 & -1 \\
0 & 0 & -2 & 0 & 2 & 1 & 1 & -1 & -1 \\
0 & 1 & -1 & 1 & -1 & 0 & 0 & 0 & 0 \\
0 & 0 & 0 & 0 & 0 & 1 & -1 & 1 & -1
\end{array}\right) .
$$

The resulting moments $\boldsymbol{m}=\boldsymbol{M f}$ are written as

$$
\boldsymbol{m}=\left(\rho, e, \varepsilon, j_{x}, q_{x}, j_{y}, q_{y}, p_{x x}, p_{x y}\right)^{T},
$$

where $e$ is related to the total energy, $\varepsilon$ is related to energy square, $\boldsymbol{j}=\rho\left(u_{x}, u_{y}\right)$ are the $\mathrm{x}$ and $\mathrm{y}$ components of the momentum, and $p_{x x}, p_{x y}$ correspond to the diagonal and offdiagonal component of the deviatoric stress tensor ${ }^{46}$, which is the traceless part of the original stress tensor. Similarly, the equilibrium distribution function $m^{e q}$ is obtained by

$$
\begin{array}{r}
\boldsymbol{m}^{e q}=\boldsymbol{M} \boldsymbol{f}^{e q}=\rho\left(1,-2+3 \boldsymbol{u}^{2}, 1-3 \boldsymbol{u}^{2},\right. \\
\left.u_{x},-u_{x}, u_{y},-u_{y}, u_{x}^{2}-u_{y}^{2}, u_{x} u_{y}\right)^{T},
\end{array}
$$

where $u_{x}$ and $u_{y}$ are the $\mathrm{x}$ and $\mathrm{y}$ components of the fluid velocity $\boldsymbol{u}$. The diagonal relaxation matrix $\mathbf{S}$ is defined as

$$
\mathbf{S}=\left(1, s_{e}, s_{\mathcal{\varepsilon}}, 1, s_{q}, 1, s_{q}, s_{V}, s_{V}\right) .
$$

The first, fourth and sixth relaxation rates correspond to density $\rho$ and momentum $\boldsymbol{j}$, they can take any values since conserved quantities do not change during collision. $s_{e}$ and $s_{v}$ are related to bulk and shear viscosities, and $s_{\varepsilon}$ and $s_{q}$ are free parameters. Following the recommendation of Ref. ${ }^{47}$, these relaxation rates are taken as $s_{e}=s_{\varepsilon}=s_{v}=1 / \tau$ and $s_{q}=8\left(2-s_{v}\right) /\left(8-s_{v}\right)$.

Based on the continuum surface force $(\mathrm{CSF})^{48}$ model, the interfacial tension can be conveniently imposed as a body force term, and it reads as

$$
\boldsymbol{F}_{s}=\frac{1}{2} \sigma K \nabla \rho^{N},
$$

where $\sigma$ is the interfacial tension coefficient, and $K$ is the local interface curvature calculated by ${ }^{48}$

$$
K=-\nabla_{s} \cdot \boldsymbol{n},
$$

where $\nabla_{s}=(\mathbf{I}-\boldsymbol{n n} \boldsymbol{n}) \cdot \boldsymbol{\nabla}$ is the surface gradient operator, $\mathbf{I}$ is the second-order identity tensor and $n=\nabla \rho^{N} /\left|\nabla \rho^{N}\right|$ is the unit normal vector. In $2 \mathrm{D}$ case, the interface curvature can be further written as

$$
K=n_{x} n_{y}\left(\frac{\partial}{\partial y} n_{x}+\frac{\partial}{\partial x} n_{y}\right)-n_{y}^{2} \frac{\partial}{\partial x} n_{x}-n_{x}^{2} \frac{\partial}{\partial y} n_{y},
$$

where $n_{x}$ and $n_{y}$ are the components of $\boldsymbol{n}$ in the $\mathrm{x}$ and y directions. In Eqs. 11 and 13, the partial derivatives are calculated using a fourth-order isotropic finite difference for minimizing the discretization errors, for example, for a variable $\psi$, its partial derivatives can be calculated by

$$
\nabla \psi(\boldsymbol{x}, t)=\frac{1}{c_{s}^{2}} \sum_{i} W_{i} \psi\left(\boldsymbol{x}+\boldsymbol{c}_{i} \delta_{t}, t\right) \boldsymbol{c}_{i} .
$$

The perturbation operator $\left(\Omega_{i}\right)^{(2)}$ is given as

$$
\left(\Omega_{i}\right)^{(2)}=\mathbf{M}^{-1}\left(\mathbf{I}-\frac{1}{2} \mathbf{S}\right) \boldsymbol{F}
$$

with

$$
\begin{aligned}
\boldsymbol{F}(\boldsymbol{x}, t) & =\left[0,6\left(u_{x} F_{s x}+u_{y} F_{s y}\right),\right. \\
& -6\left(u_{x} F_{s x}+u_{y} F_{s y}\right), F_{s x},-F_{s x}, F_{s y},-F_{s y} \\
& \left.2\left(u_{x} F_{s x}-u_{y} F_{s y}\right), u_{x} F_{s y}+u_{y} F_{s x}\right]^{T},
\end{aligned}
$$

in which $F_{s x}$ and $F_{s y}$ are the components of the body force $\boldsymbol{F}_{s}$. To correctly recover the Navier-Stokes (N-S) equations in the interfacial region where two fluids coexist the fluid velocity should be defined as ${ }^{49}$

$$
\rho \boldsymbol{u}=\sum_{i} f_{i} \boldsymbol{c}_{i}+\frac{1}{2} \boldsymbol{F}_{s} \boldsymbol{\delta}_{t}
$$

Although the perturbation operator generates the interfacial tension, the immiscibility of two fluids is not guaranteed. The recoloring step is therefore applied to promote phase segregation and maintain a sharp interface ${ }^{50}$, and is given as

$$
\left(\Omega_{i}^{R}\right)^{(3)}\left(f_{i}^{R}\right)=\frac{\rho^{R}}{\rho} f_{i}^{*}+\beta \frac{\rho^{R} \rho^{B}}{\rho^{2}} f_{i}^{e q}(\rho, \boldsymbol{u}=0) \cos \left(\varphi_{i}\right)\left|\boldsymbol{c}_{i}\right|,
$$




$$
\left(\Omega_{i}^{B}\right)^{(3)}\left(f_{i}^{B}\right)=\frac{\rho^{B}}{\rho} f_{i}^{*}-\beta \frac{\rho^{R} \rho^{B}}{\rho^{2}} f_{i}^{e q}(\rho, \boldsymbol{u}=0) \cos \left(\varphi_{i}\right)\left|\boldsymbol{c}_{i}\right|,
$$

where $f_{i}^{*}$ represents the total distribution function along the $i$-th discrete velocity direction after the perturbation and before the segregation. $\beta$ is a parameter related to the interface thickness, and can take any value between 0 and 1 to ensure positive distributions. As $\beta$ increases, the interface thickness decreases. It is chosen to be 0.7 here for numerical stability and model accuracy ${ }^{36} . \varphi_{i}$ is the angle between the indicator function gradient $\nabla \rho^{N}$ and the lattice velocity $c_{i}$, given by

$$
\cos \left(\varphi_{i}\right)=\frac{c_{i} \cdot \nabla \rho^{N}}{\left|c_{i}\right|\left|\nabla \rho^{N}\right|}
$$

\section{RESULTS AND DISCUSSION}

In this section, we will analyze the effect of various factors on the counter-current spontaneous imbibition in matrixfracture systems, which include the water injection velocity, interfacial tension, viscosity ratio, geometry configuration of the dual permeability zones and fracture spacing. In the following, we first present a detailed description of the problem, including the simulated geometries, boundary conditions, and the parameter setting. Figure 2 illustrates the primary geom-

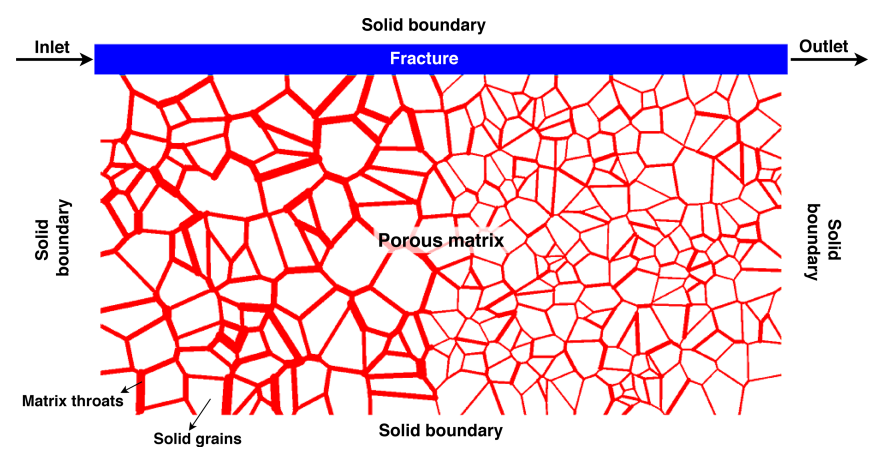

FIG. 2. Schematic of the simulated geometry used for countercurrent displacement of oil from a porous matrix by the wetting water. White color indicates the solid grains. The inlet and outlet are specified with arrows, and the matrix is initially saturated with oil (red color) and the fracture with water (blue color).

etry investigated in this study. It is composed of a rectangular porous matrix and a single fracture with the same length attached on its top side. The matrix is constructed by randomly placed throats. The throat positions are generated by applying the Voronoi tessellation technique with randomly placed sites. Details of this algorithm are described in Debnath et al. ${ }^{51}$. To introduce the heterogeneity in the matrix, the sites population density (or the number of throats) in the right-half domain is twice of that in the left-half domain. The pore diameter and throat width in both half-domains follow a truncated lognormal distribution with Lognormal $\left(\mu, \sigma^{2}\right)(\mu=0, \sigma=0.5)$, where the variation range is specified by the minimum and maximum values ${ }^{52}$. The common statistic approximation for
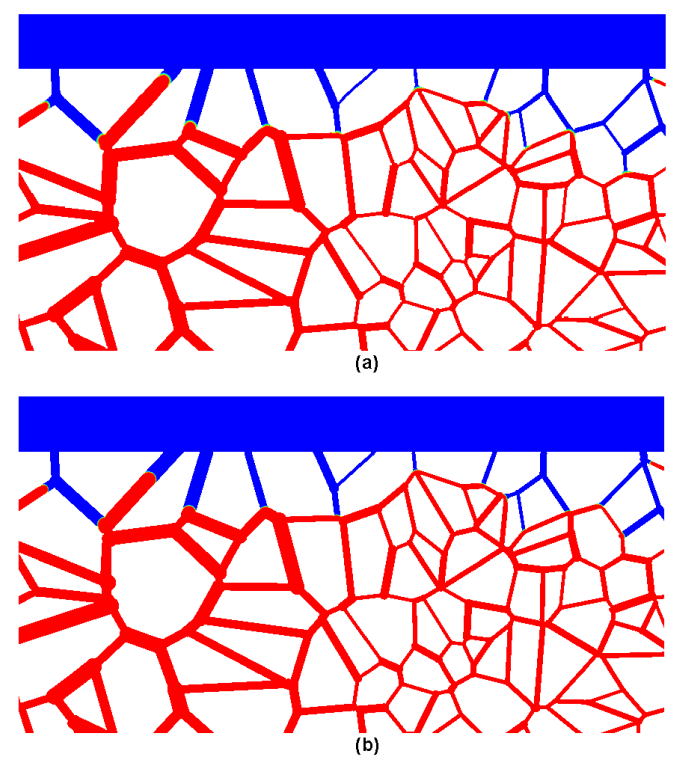

FIG. 3. Final fluid distribution in (a) the coarse grid and (b) the fine grid for $\lambda=1, \sigma=30 \mathrm{mN} / \mathrm{m}$ and $u_{i n j}=5.6 \mathrm{~mm} / \mathrm{s}$. The minimum throat width in the coarse grid is 4 lattices while in the fine grid it is 8 lattices. Note that the water and the oil are shown in blue and red respectively.

the throat widths in natural fractured porous media is the log-normal distribution ${ }^{32}$. The statistic average of the pore throat width in the left half of the domain is twice of that in the right half, yielding a dual-permeability porous matrix with high and low permeability zones. The resultant matrix porosity is 0.26 . The length and width of the computing domain is $L=13.44 \mathrm{~mm}$ and $H=7.2 \mathrm{~mm}$, which are divided into 1680 and 900 cells, respectively. The fracture width is $h=0.6 \mathrm{~mm}$ (75 lattices) and the minimum width of the pore throats is $32 \mu \mathrm{m}$ (4 lattices).

To obtain statistically meaningful results, we should apply sufficient number of lattices for the thinnest throat and simulate as many as possible pores and throats. With the restriction of the high-performance computer we can access to, we need to strike a balance on the computational efficiency and accuracy. Thus, it is important to minimize the grid number while necessary physics can be retained. Here we provide a test to examine the dependence of numerical results on the grid resolution. In order to minimize the computing resources, we take a slice of $6.888 \times 3.600 \mathrm{~mm}^{2}$ which incorporates the thinnest throats from the middle upper side of the primary geometry, i.e., "left-wide matrix" shown in Fig. 2. The simulation parameters are chosen as $\lambda=1, \sigma=30 \mathrm{mN} / \mathrm{m}$ and $u_{i n j}=5.6 \mathrm{~mm} / \mathrm{s}$ (see later for the matrix configuration and parameter setting). Fig. 3(a) shows the final fluid distribution in the coarse grid with the thinnest throat width of 4 lattices and (b) shows the final fluid distribution in the fine grid with the thinnest throat width of 8 lattices. It is observed that the grid refinement only slightly affects the numerical results. The final oil saturation in the matrix varies from 0.114 in the coarse 
grid to 0.113 in the fine grid, with a difference of $0.88 \%$. This verifies that the coarse grid simulation with the thinnest throat width of 4 lattices can offer acceptable computational accuracy.

Initially, the fracture and the matrix are saturated with water and oil (denoted by blue and red in Fig. 2), respectively. Then water is continuously injected from the left inlet of the fracture with a constant velocity of $u_{\mathrm{inj}}$, and the outlet pressure is set to be zero. Both water and the expelled oil flow out at the right end of the fracture. All the sides of the matrix are assumed to be solid walls, except for the top side, which is connected to the fracture. At the solid surfaces, no-slip boundary condition is imposed by using the half-way bounceback scheme $\mathrm{e}^{53}$ and the desired contact angle is achieved by using the wetting boundary condition proposed by $\mathrm{Xu}$ et al. ${ }^{24}$, which modifies the direction of the colour gradient $\nabla \rho^{N}$ at the boundary to match the specified contact angle $\theta$. These boundary conditions are to maintain a counter-current imbibition environment in which the oil in the matrix can only flow into the fracture that supplies water, which have been widely adopted in the previous numerical and experimental studies of counter-current imbibition ${ }^{4,9,23,54}$. Among various factors that influence the capillary imbibition, surface wettability is of vital importance for both imbibition rate and ultimate oil recovery, and its effect has been thoroughly investigated by Rokhforouz and Amiri ${ }^{4}$. They found that, when the contact angle $\theta$ (measured from the water side) is greater than $\pi / 4$, the oil recovery is negligible, but both the imbibition rate and the oil recovery drastically increase with decreasing contact angle when $\theta<\pi / 4$. Similar findings are also demonstrated by the present simulations and the simulation results are shown in Appendix B, where the effect of surface wettability is studied for the contact angles varying from $\pi / 10$ to $\pi / 4$. Therefore, the porous matrix is considered strongly water-wet with $\theta=\pi / 10$ in the following study.

For most pore-scale studies, the ratio of gravity to capillary forces is very small and thus the effects of density difference can be neglected ${ }^{55}$. In the LB simulations, the densities of both fluids are set to unity for the sake of simplicity. Five different water-oil viscosity ratios are considered, i.e. $\lambda=0.1,0.5,1,5$ and 10 , and six different interface tensions are used, i.e. $\sigma=5,10,15,30,45$ and $60 \mathrm{mN} / \mathrm{m}$. The water injection velocity at the inlet ranges from $u_{\text {inj }}=0.075 \mathrm{~mm} / \mathrm{s}$ to $44.4 \mathrm{~mm} / \mathrm{s}$. Unless otherwise stated, the simulation parameters are chosen as $\lambda=1, \sigma=30 \mathrm{mN} / \mathrm{m}$ and $u_{\text {inj }}=5.6 \mathrm{~mm} / \mathrm{s}$. For each case, the simulation was run until the saturation of water in the matrix reached a constant value.

Apart from the primary geometry setup as illustrated in Fig. 2, we also consider several different geometries that are derived from it to study the effect of matrix heterogeneity and the interplay between imbibition and fluid transport in the fractures. The first variant is the mirrored one by reversing the matrix from left to right. The second variant is the twofracture system by adding an additional horizontal fracture at various positions.

\section{A. Effect of water injection velocity}

The interaction of water with the matrix is strongly related to the rate of water injection ${ }^{56}$ as the expelled oil accumulates and transports in the fracture. In order to investigate the effect of water injection velocity, eight numerical experiments with the injection velocities of $0.075,0.11,0.56,1.11,4.44,5.56$, 22.22 and $44.44 \mathrm{~mm} / \mathrm{s}$ are conducted.

Figure 4 shows snapshots of the fluid distributions for various injection velocities. It is observed that regardless of the water injection velocity, the oil selects the widest matrix throat connecting with the fracture to flow into the fracture, while water in the fracture imbibes into the matrix from the other narrower matrix throats. This is because the wider throat corresponding to the lower capillary pressure makes the water imbibition more difficult. Such a pattern was also observed by Gunde et al. ${ }^{23}$ when they analyzed the counter-current imbibition in a fracture-matrix system where the porous matrix is composed of randomly placed square solid grains.

As more oil comes out of the matrix from the widest channel continuously, the oil accumulates locally to form either piston-like plug, elongated liquid thread, or isolated drops, depending on the water injection velocity. The water imbibition characteristics including the preferential path and the imbibition depth also change significantly with the injection velocity. We will later discuss these differences from the perspective of different regimes.

When the oil cannot further move out from the matrix, we assume the imbibition has reached the steady state. The fluid distributions at the steady state for the eight different injection velocities are presented in Fig. 5. It shows the oil recovery factor and the imbibition depth overall increase as the water injection velocity decreases. For the present geometry, one would expect that the wetting fluid (water) will preferentially enter the narrowest neighboring throat due to its highest capillary pressure ${ }^{57}$. Such a trend is observed in the early stage, while in the late stage, the water front could invade the matrix further in the left-half region where the throat width is larger [see Fig. 5(c)-(f)]. Again, this will be discussed in more details later.

Based on the different morphologies of the expelled oil in the fracture, as shown in Fig. 4, we identified three different regimes: the squeezing regime when the expelled oil forms a piston-like plug and grows in the fracture [Fig. 4 (a) - (d)]; the dripping regime when the expelled oil forms isolated drops in the fracture [Fig. $4(\mathrm{~g})$ and $(\mathrm{h})$ ]; and the jetting regime where the expelled oil develops into a thin and elongated oil thread [Fig. 4 (e) and (f)]. It is noted that a similar regime classification was also performed in the studies of droplet formation using a microfluidic T-junction ${ }^{58-60}$. These three different regimes are analyzed below individually.

First, when the water injection velocity is small (typically varying from $0.075 \mathrm{~mm} / \mathrm{s}$ to $1.11 \mathrm{~mm} / \mathrm{s}$ ), the squeezing regime occurs, where the expelled oil blobs form a continually growing plug in the fracture [Fig. 4 (a) - (d)]. The oil plug is big enough to entirely block water flowing through the fracture. As a result, the pressure builds up at the upstream of the oil plug as observed in the simulations. The increased 

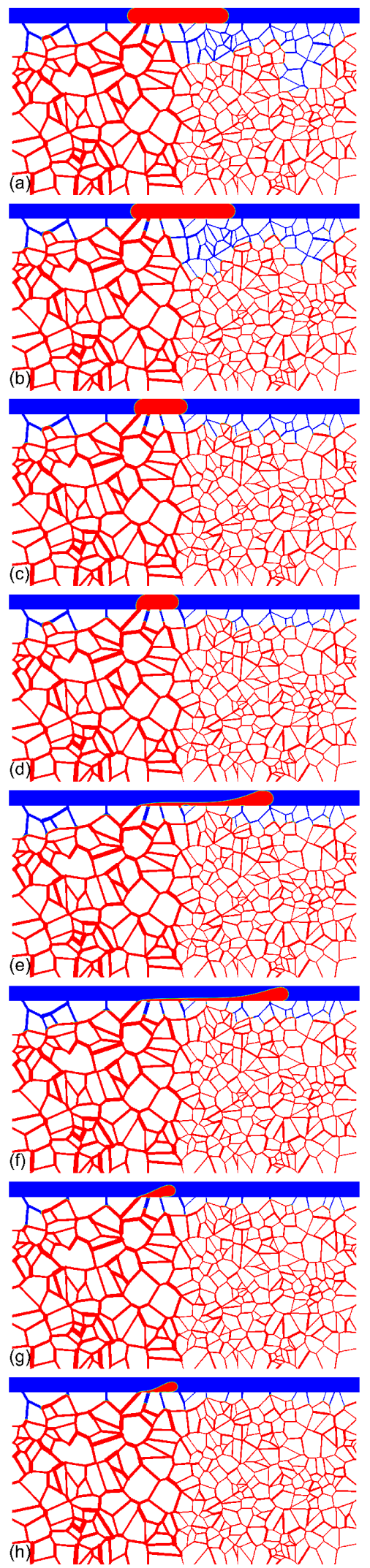
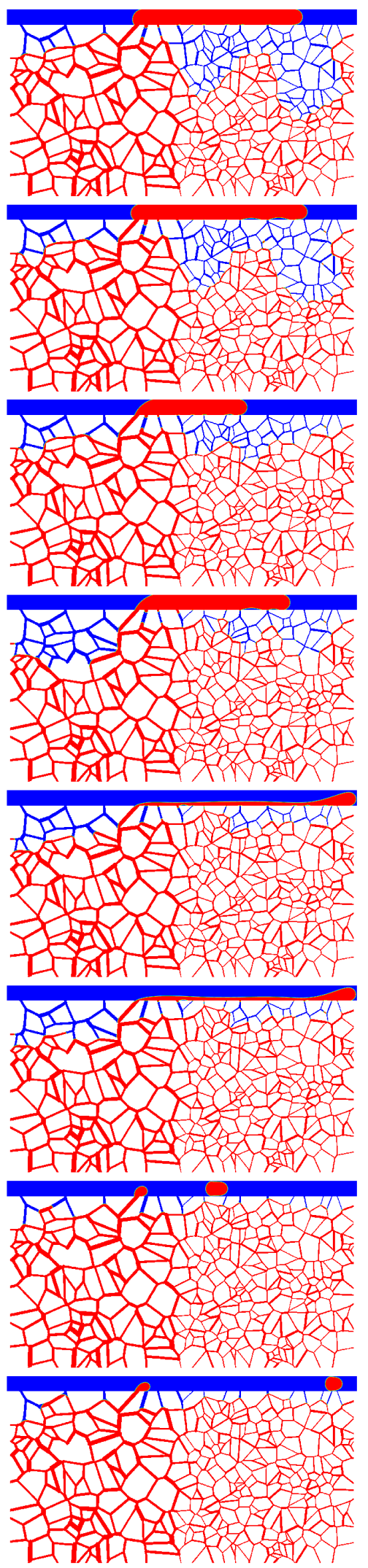
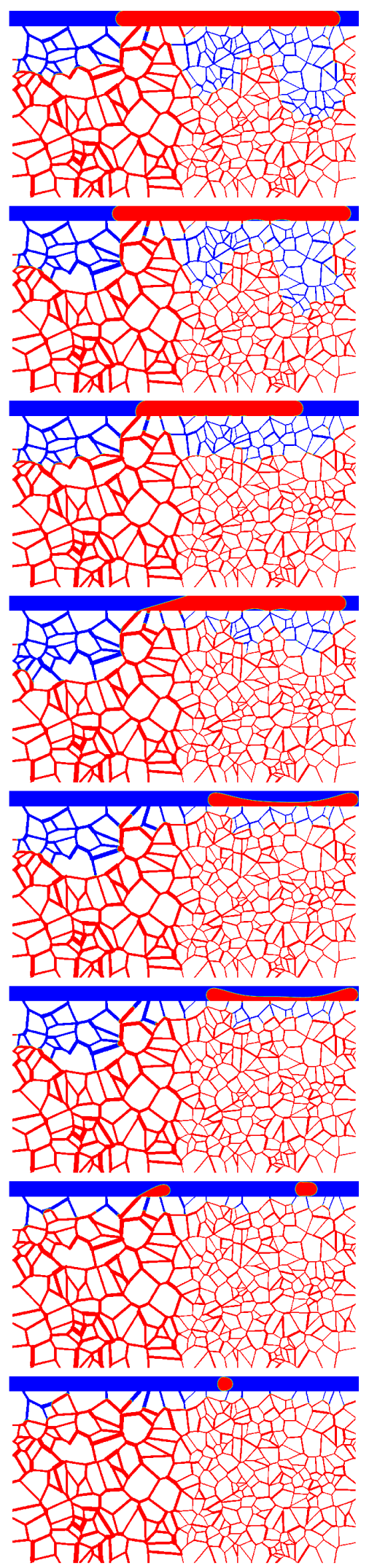

FIG. 4. Snapshots of fluid distributions during imbibition for different injection velocities: (a) $u_{\text {inj }}=0.075 \mathrm{~mm} / \mathrm{s} ;$ (b) $u_{\text {inj }}=0.11 \mathrm{~mm} / \mathrm{s}$; (c) $u_{\text {inj }}=0.56 \mathrm{~mm} / \mathrm{s}$; (d) $u_{\text {inj }}=1.11 \mathrm{~mm} / \mathrm{s}$; (e) $u_{\text {inj }}=4.44 \mathrm{~mm} / \mathrm{s}$; (f) $u_{\text {inj }}=5.56 \mathrm{~mm} / \mathrm{s} ;(\mathrm{g}) u_{\text {inj }}=22.22 \mathrm{~mm} / \mathrm{s}$ and (h) $u_{\text {inj }}=44.44 \mathrm{~mm} / \mathrm{s}$. Note that the water and the oil are shown in blue and red respectively. 


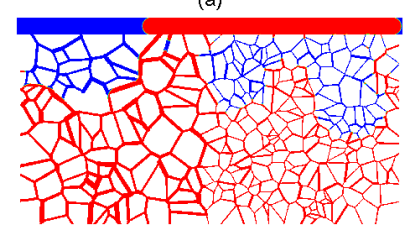

(e)

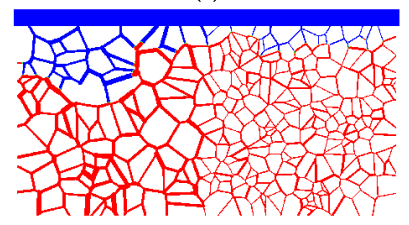

(b)

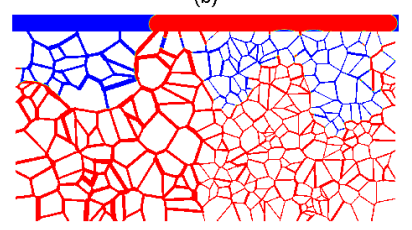

(f)

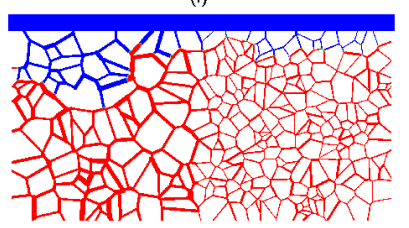

(c)

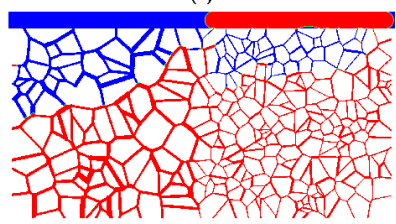

(g)

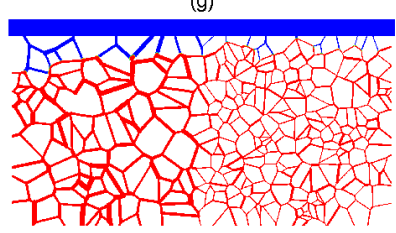

(d)

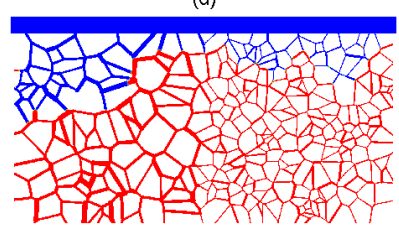

(h)

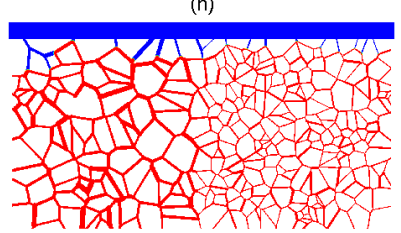

FIG. 5. Fluid distributions at steady-state for different injection velocities: (a) $u_{\text {inj }}=0.075 \mathrm{~mm} / \mathrm{s} ;$ (b) $u_{\text {inj }}=0.11 \mathrm{~mm} / \mathrm{s} ;(\mathrm{c}) u_{\text {inj }}=0.56 \mathrm{~mm} / \mathrm{s}$; (d) $u_{\text {inj }}=1.11 \mathrm{~mm} / \mathrm{s}$; (e) $u_{\text {inj }}=4.44 \mathrm{~mm} / \mathrm{s}$; (f) $u_{\text {inj }}=5.56 \mathrm{~mm} / \mathrm{s} ;(\mathrm{g}) u_{\text {inj }}=22.22 \mathrm{~mm} / \mathrm{s}$ and $(\mathrm{h}) u_{\text {inj }}=44.44 \mathrm{~mm} / \mathrm{s}$. Note that the water and the oil are shown in blue and red respectively.

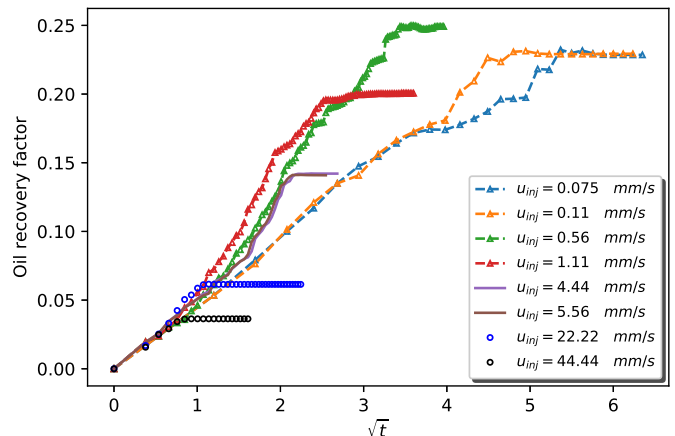

(a)

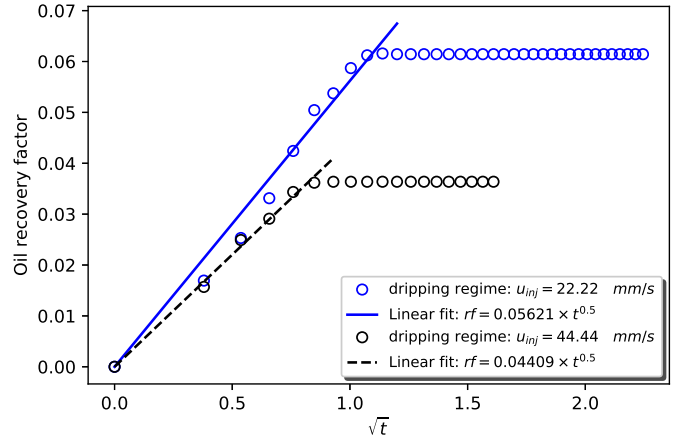

(b)

FIG. 6. (a) Time evolution of the oil recovery factor for different injection velocities of $0.075 \mathrm{~mm} / \mathrm{s}, 0.11 \mathrm{~mm} / \mathrm{s}, 0.56 \mathrm{~mm} / \mathrm{s}, 1.11 \mathrm{~mm} / \mathrm{s}$, $4.44 \mathrm{~mm} / \mathrm{s}, 5.56 \mathrm{~mm} / \mathrm{s}, 22.22 \mathrm{~mm} / \mathrm{s}$ and $44.44 \mathrm{~mm} / \mathrm{s}$; (b) The magnified illustration of the oil recovery factor evolution in the dripping regime.

pressure at the upstream forces water to advance into the lefthalf region of the matrix gradually from the upstream. At the same time, as the plug length increases, it blocks the imbibition of water into the narrower throats in the right-half region of the matrix. This mechanism explains why we observe the counterintuitive phenomenon that water advances further in the left-half region of the matrix which has wider throats, as shown in Fig. 5(c) and (d). For the extremely low water injection rate, e.g. $u_{\text {inj }} \leq 0.11 \mathrm{~mm} / \mathrm{s}$, we can clearly see from Fig. 5(a) and (b) that water progresses with three distinctive capillary fingers into the matrix. In the squeezing regime, thanks to the blocking effect from the oil plug, more water imbibes into the matrix and thus a relatively large amount of oil is expelled from the matrix into the fracture. This suggests a higher oil recovery factor at steady state as shown in Fig. 6(a).

As the water injection velocity increases further, e.g., $u_{\mathrm{inj}}=$ $4.44 \mathrm{~mm} / \mathrm{s}$ and $5.56 \mathrm{~mm} / \mathrm{s}$, the jetting regime occurs where the expelled oil exhibits a shape different from the one in the squeezing regime. Due to the higher water injection velocity in the fracture, the oil droplet is stretched into a long thread by the incoming water, with its root attached to the widest matrix channel that supplies the expelled oil [Fig. 4 (e) and (f)]. After the oil thread gets pinched off at its root, it quickly retracts to a dumbbell shape. During the early stage of the oil thread spreading towards the right end of the fracture, the imbibition of water into the right-half region of the matrix is quickly blocked, which explains why the final oil recovery factor is much less than that in the squeezing regime [see Fig. 6(a)]. The dumbbell-shaped oil thread eventually flows out of the fracture, which can be seen from Fig. 5 (e) and (f).

As the rate of water injection continues to increase, the expelled oil enters the fracture as isolated drops [Fig. 4 (g) and (h)], which is known as the dripping regime. The higher injection velocity results in a larger shear force acting on the expelled oil drop, leading to an earlier pinch-off before it can grow longer. The size of the drop decreases with increasing water injection rate. These drops are transported quickly along the fracture by the injected water. Since the water is able to freely flow out through the fracture and no blocking effect 


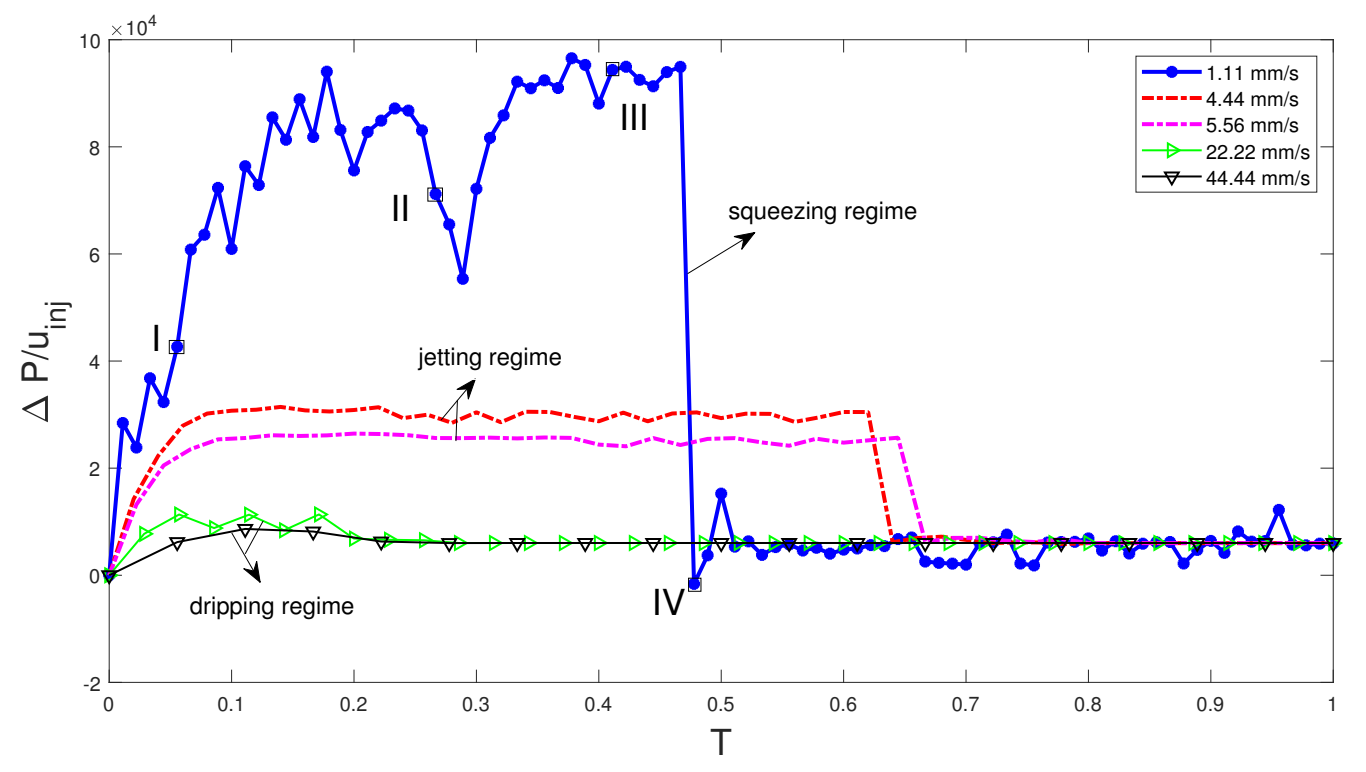

FIG. 7. Time evolution of the pressure difference (which is scaled against the water injection velocity $u_{\text {inj }}$ ) between the upstream and outlet in the fracture for three typical regimes. Blue lines with solid circles correspond to the case shown in Fig. 4(d) (the squeezing regime), the dashed red and magenta lines correspond to Fig. 4(e) and (f) (the jetting regime), and the green and black lines with open triangles correspond to Fig. 4(g) and (h) (the dripping regime). The black squares labelled I, II and III correspond to the three snapshots shown in Fig. 4(d), while IV represents the instant at which the widest matrix throat connected with the fracture being invaded by water. The dimensionless time $T$ is defined by $T=t / t_{s}$ where $t_{s}$ is the time of reaching the steady state. The values of $t_{s}$ are $12.96 \mathrm{~s}, 7.2 \mathrm{~s}, 6.48 \mathrm{~s}, 5.04 \mathrm{~s}$ and $2.59 \mathrm{~s}$ for Fig. $4(\mathrm{~d})-(\mathrm{h})$.
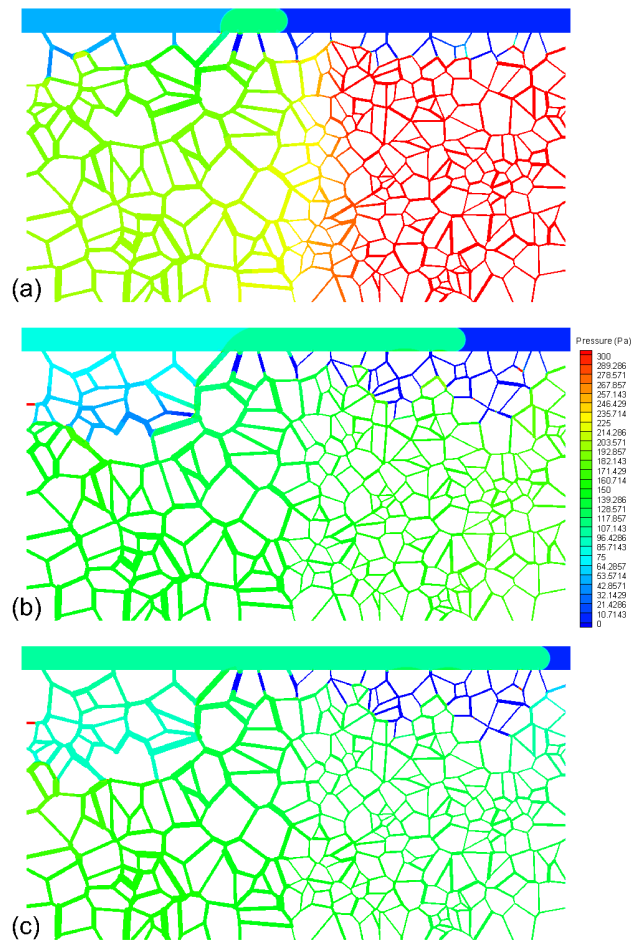

FIG. 8. The distributions of pressure (difference to the outlet pressure) at the times of (a) $t=0.72 \mathrm{~s}$; (b) $t=3.46 \mathrm{~s}$ and (c) $t=5.33 \mathrm{~s}$, which correspond to the three snapshots shown in Fig. 4(d). exists, overall imbibition depth is much shallower than that in the other two regimes. Accordingly, the oil recovery factor in this regime is the lowest among all the three regimes, typically lower than $8 \%$ as observed in Fig. 6(a). On the other hand, the water infiltration path into the matrix is less selective than the other two regimes, as can been seen from Fig. $5(\mathrm{~g})$ and $(\mathrm{h})$ where the imbibition front progresses almost uniformly down into the matrix. This regime resembles the "instantly filled fracture" regime identified experimentally by Rangel-German et al. ${ }^{21}$, in which water is mainly pumped through the fracture and little water is imbibed into the matrix. In addition, the water saturation in the matrix in this regime scales linearly (correlation coefficient of 0.99) with the square root of time before reaching the steady state [see Fig. 6(b)], which is consistent with the experimental finding of Rangel-German et al. ${ }^{56}$.

Among the above three regimes, the squeezing regime has the highest oil recovery factor and takes a relatively long time to reach the steady state. Specifically, the maximum oil recovery factor $\mathrm{rf}=0.25$ is reached at $u_{\mathrm{inj}}=0.56 \mathrm{~mm} / \mathrm{s}$ in the squeezing regime. On the contrary, in the dripping regime, the imbibition reaches the steady state almost instantly, and the final oil recovery factor is extremely low. We also record the pressure differences between the upstream and outlet in the fracture during the displacement for the three typical regimes, and the results are shown in Fig. 7. Among these three regimes, the pressure build-up is the most significant in the squeezing regime. For the squeezing regime, the pressure upstream keeps at a high level before reaching IV, although sudden drop occurs when more wetting fluid is imbibed into 

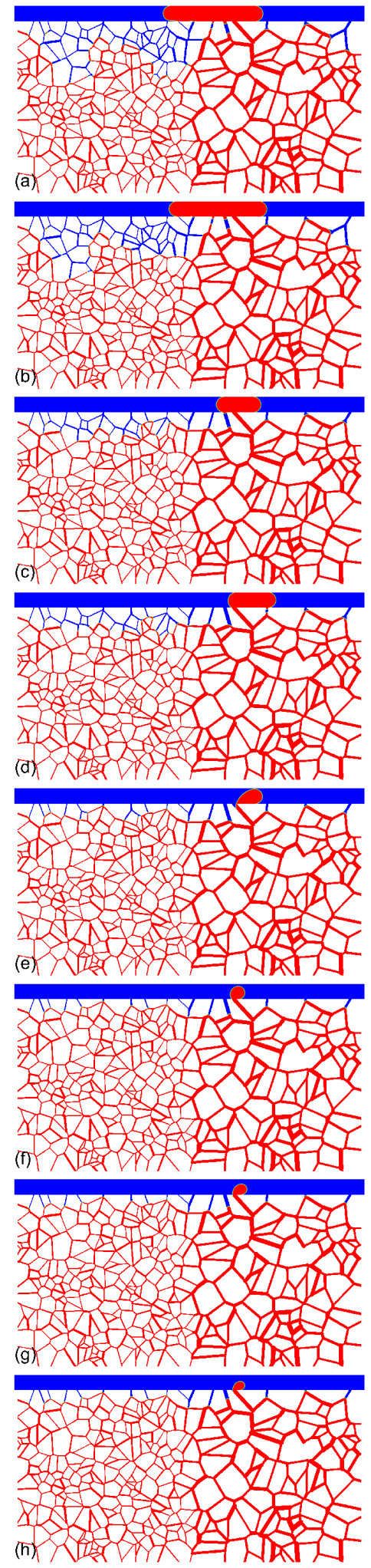
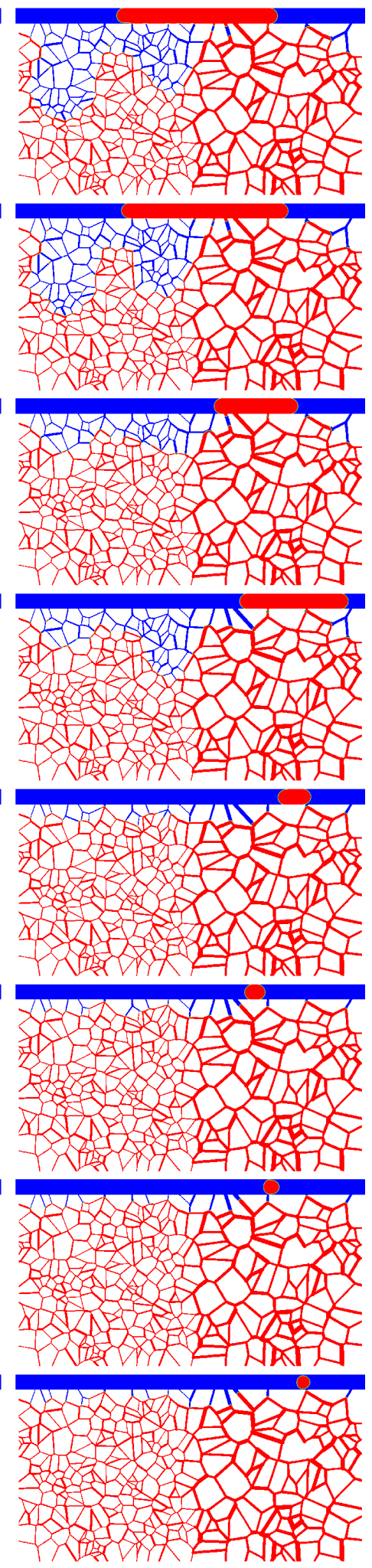
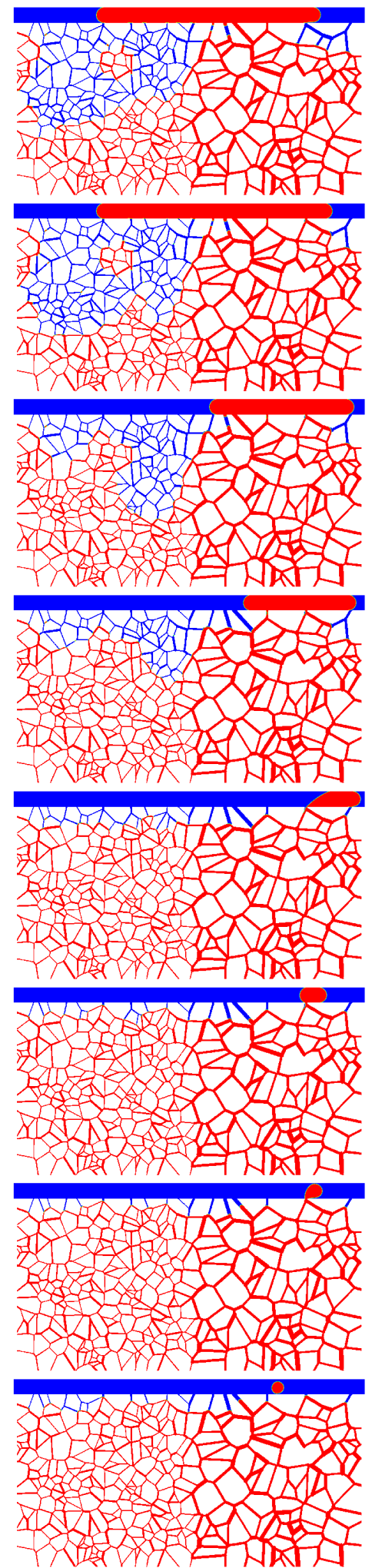

FIG. 9. Snapshots of fluid distributions during imbibition in the right-wide matrix for different injection velocities: (a) $u_{\text {inj }}=0.075 \mathrm{~mm} / \mathrm{s}$;

(b) $u_{\text {inj }}=0.11 \mathrm{~mm} / \mathrm{s}$; (c) $u_{\text {inj }}=0.56 \mathrm{~mm} / \mathrm{s} ;$ (d) $u_{\text {inj }}=1.11 \mathrm{~mm} / \mathrm{s}$; (e) $u_{\text {inj }}=4.44 \mathrm{~mm} / \mathrm{s}$; (f) $u_{\text {inj }}=5.56 \mathrm{~mm} / \mathrm{s} ;$ (g) $u_{\text {inj }}=22.22 \mathrm{~mm} / \mathrm{s}$ and (h) $u_{\mathrm{inj}}=44.44 \mathrm{~mm} / \mathrm{s}$. Note that the water and the oil are shown in blue and red respectively. 
(a)

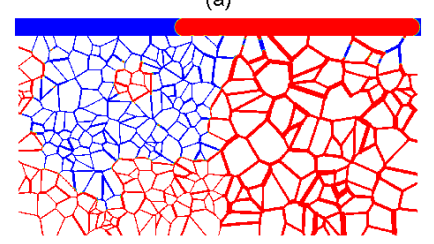

(e)

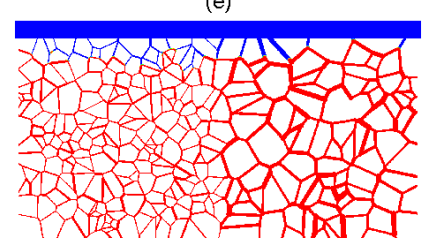

(b)

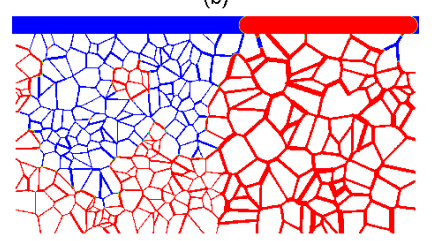

(f)

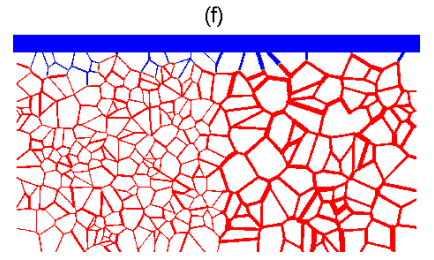

(c)

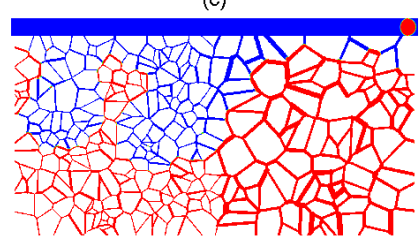

(g)

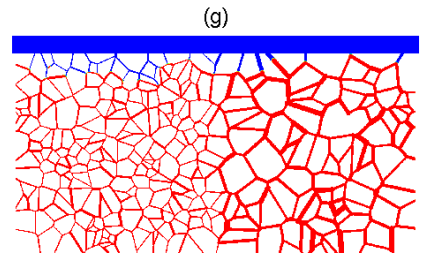

(d)

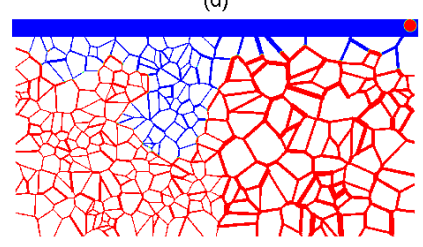

(h)

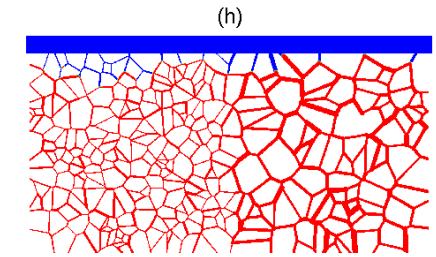

FIG. 10. Steady-state fluid distributions in the right-wide matrix for different injection velocities: (a) $u_{\text {inj }}=0.075 \mathrm{~mm} / \mathrm{s} ;(\mathrm{b}) u_{\text {inj }}=0.11 \mathrm{~mm} / \mathrm{s}$; (c) $u_{\text {inj }}=0.56 \mathrm{~mm} / \mathrm{s}$; (d) $u_{\text {inj }}=1.11 \mathrm{~mm} / \mathrm{s}$; (e) $u_{\text {inj }}=4.44 \mathrm{~mm} / \mathrm{s}$; (f) $u_{\text {inj }}=5.56 \mathrm{~mm} / \mathrm{s} ;(\mathrm{g}) u_{\text {inj }}=22.22 \mathrm{~mm} / \mathrm{s}$ and (h) $u_{\text {inj }}=44.44 \mathrm{~mm} / \mathrm{s}$. Note that the water and the oil are shown in blue and red respectively.

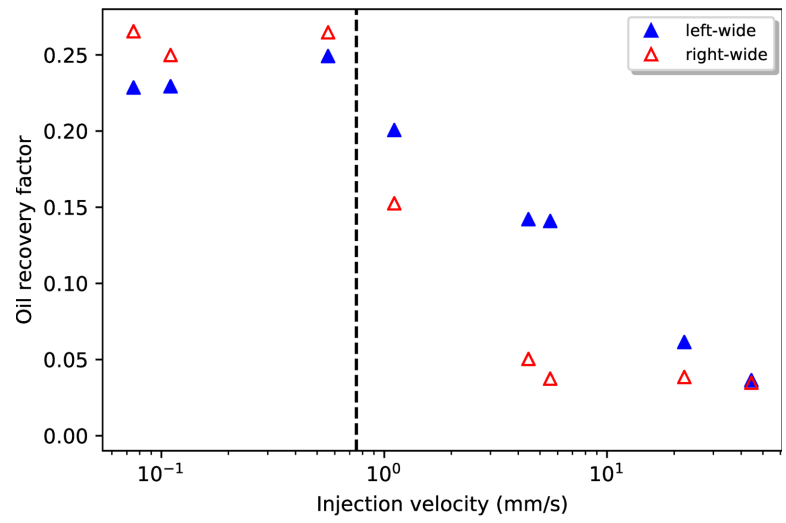

FIG. 11. Ultimate oil recovery factor as a function of injection velocity $u_{\text {inj }}$ for both the left-wide and the right-wide matrixes.

the matrix. The pressure build-up process in the squeezing regime can be also seen from the pressure distributions plotted in Fig. 8. As the oil plug grows, the pressure upstream in the fracture increases while the pressure of the non-wetting fluid in the matrix decreases [Fig. 8(a)-(c)]. For the jetting regime, a certain level of pressure build-up is also observed, which is consistent with the fluid distributions in Fig. 4(e) and (f). In contrast, the pressure upstream maintains at a rather low level throughout the displacement process for the dripping regime.

\section{B. Effect of geometry configuration of the dual permeability zones}

The porous matrix in the primary geometry is intentionally constructed as two different regions, where the mean channel width in the left-half region of the matrix is twice of that in the right-half region. Such an geometry configuration of the matrix is expected to significantly influence the water imbibition process due to the interplay of the water imbibition into the matrix and oil accumulation and transport in the fracture. As previously shown in Fig. 4, the oil blobs prefer flowing through the widest throat into the fracture and accumulate locally in the fracture towards the downstream direction. In the squeezing and jetting regimes, the oil plug or thread would entirely or partially block the matrix entrances downstream. Thus, it is important whether the high permeability zone is located upstream or downstream of the fracture.

In this subsection, we investigate how the geometry configuration of the high and low permeability zones affects the imbibition process and the oil recovery factor. By reversing the matrix in the primary geometry (Fig. 2) from left to right, we create another geometry with wide throats distributed in the right-half region of the matrix and the water is still injected from the left end of the fracture. The new set-up is tantamount to reversing the water injection direction in Fig. 2. We now distinguish the two matrixes by calling the original one "leftwide matrix" and the new one "right-wide matrix", respectively. The left-wide matrix corresponds to the geometry configuration that the high permeability zone is located upstream and the low permeability zone downstream. By contrast, the right-wide matrix has the high permeability zone downstream and the low permeability zone upstream.

Fig. 9 shows the water imbibition process in the right-wide matrix and the dynamics of expelled oil blobs in the fracture for eight different injection velocities. Note that the corresponding results regarding the left-wide matrix have been shown in Fig. 4. Different from the results in Fig. 4, it is seen in Fig. 9 that the squeezing regime [Fig. 9(a-d)] changes to the dripping regime [Fig. 9(e-h)] directly without undergoing the jetting regime, as the oil shape in the fracture changes from plug to small drop by increasing injection velocity. The regime alteration process is seen clearly from row (d) to (e) in Fig. 9, as the location of the high permeability zone is downstream near the outlet of the fracture, the expelled oil blobs from the widest throat are snapped off easily and carried away quickly as the injection velocity increases. Thus the elongation of the oil thread is less likely to happen. In ad- 
dition, we also notice that water advances almost exclusively into the matrix from the left-half region, i.e. low permeability zone [also see the steady-state fluid distributions in Fig. 10 (a)-(d)], whereas in the left-wide matrix, water invades mostly into the high permeability zone. This difference lies in that in the right-wide matrix, the preference of water imbibition into the narrower throats is attributed not only to the larger capillary pressure, but also to the build-up squeezing pressure.

Figure 11 plots the final oil recovery factor as a function of water injection velocity for both the left-wide matrix and the right-wide matrix. It reveals that the oil recovery factor is high (around $0.22 \sim 0.26)$ at low injection velocity $\left(u_{\text {inj }} \leq 0.78\right.$ $\mathrm{mm} / \mathrm{s}$ ) in both geometry configurations. Upon increasing $u_{\text {inj }}$ from $1.11 \mathrm{~mm} / \mathrm{s}$, the oil recovery factor decreases and reaches its minimum value of 0.04 at the highest injection velocity in both matrix geometries. It is seen in Fig. 11 that there exists a critical velocity (around $0.78 \mathrm{~mm} / \mathrm{s}$ ) above which the leftwide matrix has a higher oil recovery factor, but below which, the right-wide matrix has a higher oil recovery factor. In the left-wide geometry, water is found to advance further into the left-half region (high permeability zone) which is connected to the upstream of the fracture. This counters our intuition that wider throats corresponding to smaller capillary pressure unfavor the imbibition of water into the matrix. We speculate that this is due to the build-up squeezing pressure upstream when the oil blobs accumulate at the downstream of the fracture and block the fracture channel. By flipping the geometry from "left-wide matrix" to "right-wide matrix", we again observe that water invades deeper into the left-half region of the matrix (low permeability zone) that is adjacent to the upstream of the fracture, which confirms our speculation. To conclude, the geometry configuration of the dual permeability zones plays a significant role in the oil recovery by countercurrent imbibition in a fracture-matrix system.

\section{Effect of interfacial tension}

As spontaneous imbibition is a result of capillary pressure, the dynamic imbibition process and the final recovery factor are significantly affected by the interfacial tension between two fluids ${ }^{61}$. To investigate the impact of the interfacial tension on the oil recovery process in a fracture-matrix system, six different simulations with the interfacial tension values of $\sigma=5,10,15,30,45$ and $60 \mathrm{mN} / \mathrm{m}$ are carried out. All the other parameters are chosen as the default values mentioned before, and the primary geometry, i.e. the left-wide matrix is used as an illustration.

According to the Young-Laplace equation, the capillary intrusion of water into the matrix is weakened with the decrease of interfacial tension $\sigma$. This is confirmed by the imbibition depths in Fig. 12, which shows the dynamic imbibition processes at different values of $\sigma$. It is also observed that with the interfacial tension decreasing from $30 \mathrm{mN} / \mathrm{m}$ to $5 \mathrm{mN} / \mathrm{m}$, the flow regime for the expelled oil changes from the jetting regime [Fig. 12 (a) and (b)] to the dripping regime [Fig. 12 (c)]. In this regard, decreasing interfacial tension has a similar effect to increasing water injection velocity, which can be explained by the competition between the viscous shear force exerted on the expelled oil blobs and the capillary force resisting the interface deformation. However, the imbibition behavior in the matrix cannot be described by the capillary number (Ca). The capillary number is often used to characterize the forced displacement, in which the characteristic velocity, as a measure of viscous force, is defined as the injection velocity or the imposed pressure difference. In the spontaneous imbibition, the imbibition rate is not determined by the injection velocity of water into the fracture but by the interfacial tension, suggesting that it is more appropriate to use the interfacial tension rather than the capillary number defined through the injection velocity of water for our discussion.

Figure 13 presents the steady-state oil-water distribution for different interfacial tensions. It shows that with larger interfacial tension, the water imbibes deeper into the left-half matrix with high permeability. This is because the elongated expelled oil in the jetting regime can block the fracture channel to some extent, leading to an increased upstream pressure that drives more water into the high permeability zone. In addition, increasing $\sigma$ will increase the capillary pressure, which acts as the only driving force for spontaneous imbibition of water into the matrix, leading to more water imbibed into the matrix and enhancing the oil recovery.

Figure 14 displays the oil recovery factor as a function of the square root of time for different interfacial tensions. It is found that a larger interfacial tension leads to a higher imbibition rate and usually a higher ultimate oil recovery factor. These trends are consistent with the previous findings by Rokhforouz and Amiri ${ }^{4}$ who studied the imbibition of water from a fracture into a porous media composed of randomly distributed cylinders using the phase-field method. In addition, Fig. 14 shows that in the dripping regime, the linear relationship between the oil recovery factor and the square root of time is still valid.

\section{Effect of viscosity ratio}

The effect of viscosity ratio, defined as $\lambda=\mu_{\text {water }} / \mu_{\text {oil }}$, is investigated for $\lambda=0.1,0.5,1,5$ and 10 . Different values of viscosity ratio are achieved by adjusting the viscosity of oil while keeping the viscosity of water unchanged.

Fig. 15(a) shows snapshots of the fluid distributions for the viscosity ratios of $0.1,1$ and 10 . It is found in the fracture that the expelled oil morphology changes from jetting regime to the dripping regime with increasing viscosity ratio. In the jetting regime, see Fig. 15 [row (a)], water progresses more in the high permeability zone because the long oil thread blocks the water passage into the low permeability zone. Fig. 15(b) shows a combination of the jetting and dripping regimes. Clearly, water advances into the matrix within a very limited depth because of high viscous force between oil and the matrix wall. When the viscosity ratio is increased to 10, see Fig. 15(c), the oil blob occupies the whole crosssection of the fracture, and the squeezing regime seemingly occurs. However, the squeezing pressure upstream is not built up because the oil plugs in the fracture are driven by water 

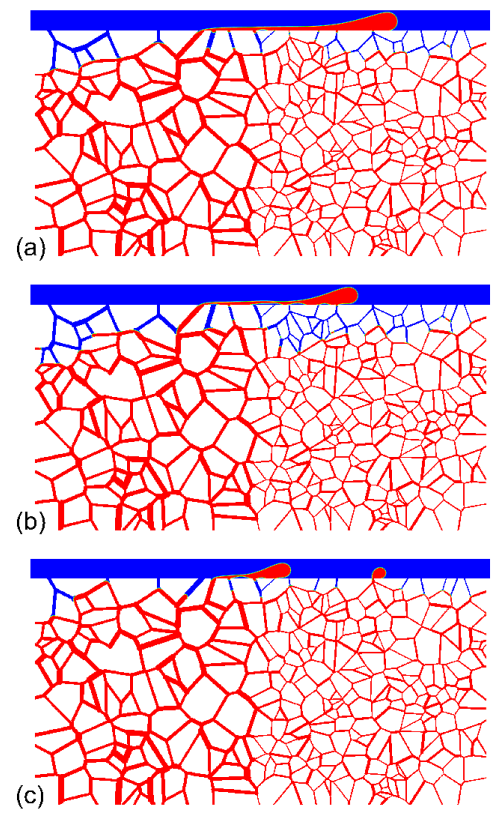
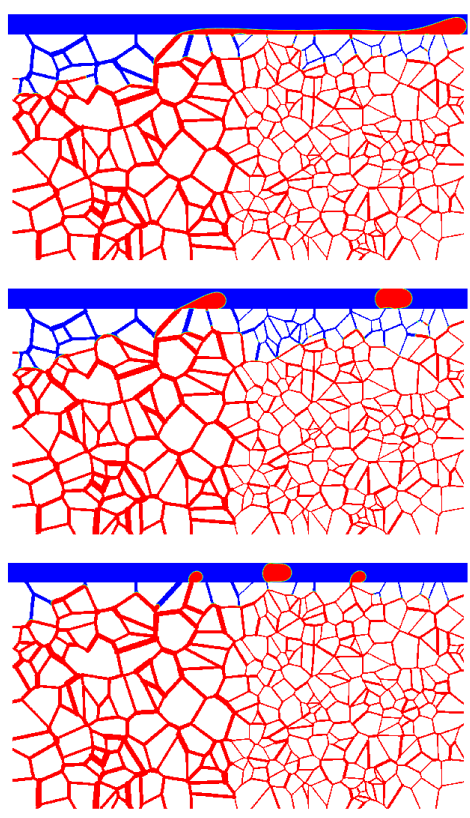
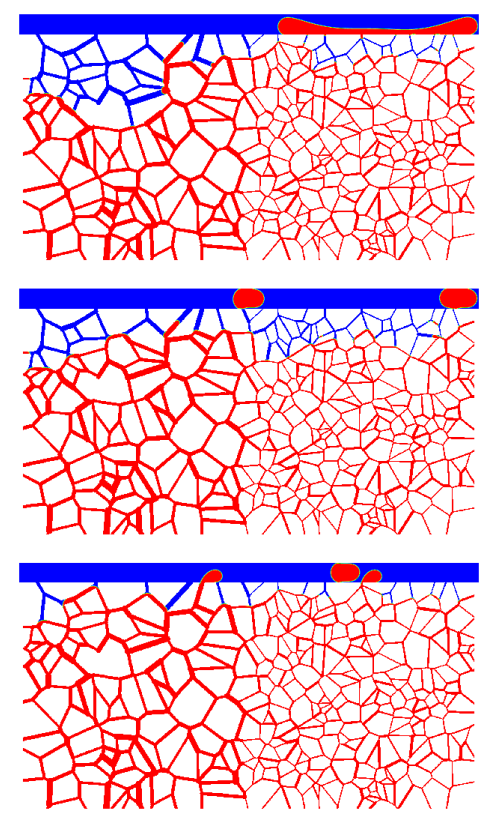

FIG. 12. Snapshots of fluid distributions during imbibition for different interfacial tensions: (a) $\sigma=30 \mathrm{mN} / \mathrm{m}$; (b) $\sigma=15 \mathrm{mN} / \mathrm{m}$ and (c) $\sigma=5 \mathrm{mN} / \mathrm{m}$. Note that the water and the oil are shown in blue and red respectively.

(a)

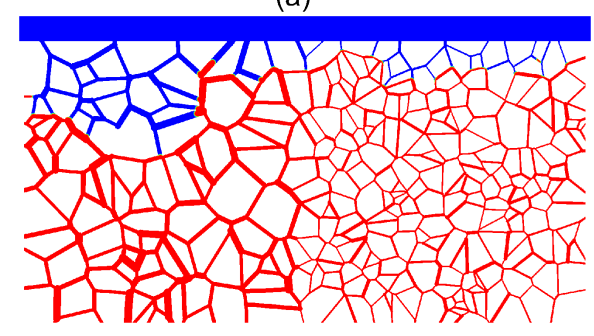

(b)

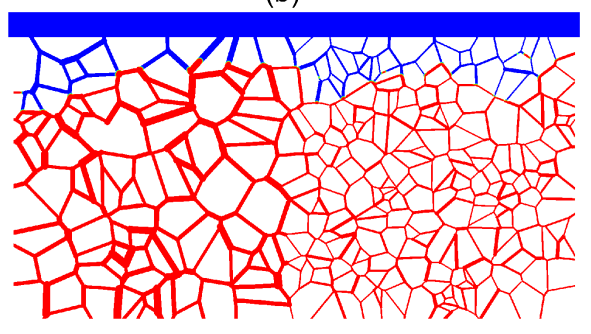

(c)

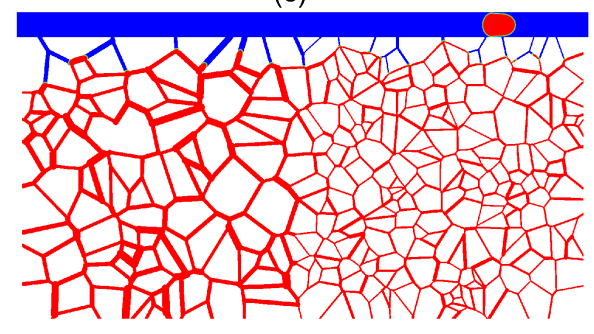

FIG. 13. Fluid distributions at steady-state for different interfacial tensions: (a) $\sigma=30 \mathrm{mN} / \mathrm{m}$; (b) $\sigma=15 \mathrm{mN} / \mathrm{m}$ and (c) $\sigma=5 \mathrm{mN} / \mathrm{m}$. Note that the water and the oil are shown in blue and red respectively.

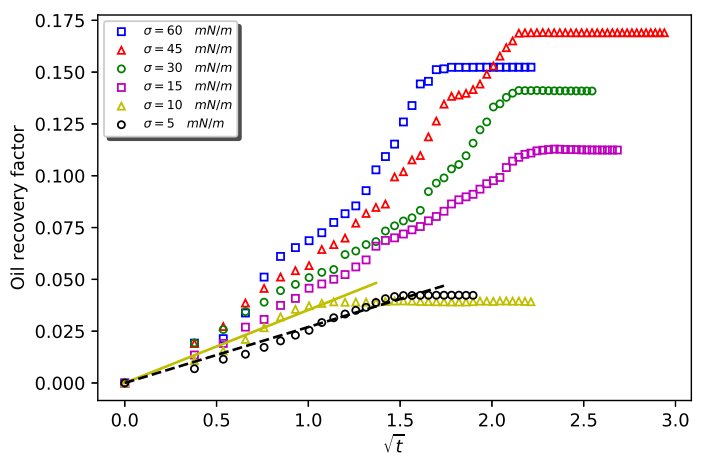

FIG. 14. Time evolution of oil recovery factor for various water-oil interfacial tensions. The solid and dashed lines are the linear fits to the data of $\sigma=10 \mathrm{mN} / \mathrm{m}$ and $\sigma=5 \mathrm{mN} / \mathrm{m}$, respectively, which are given by $r f=0.03524 \times t^{0.5}$ and $r f=0.02699 \times t^{0.5}$. towards the outlet quickly. Note that the formed oil plugs move more easily towards the outlet in the fracture when the oil phase is of lower viscosity. In this case, the water front advances evenly into the left and right regions of the matrix like in the dripping regime. Figure 16 displays the oil recovery factor as a function of the square root of time for various viscosity ratios. It is clear that the highest oil recovery factor is reached when $\lambda=0.5$, different from the previous finding of Rokhforouz and Amiri ${ }^{4}$, who found the highest oil recovery factor occurring at the viscosity ratio of unity. In addition, the imbibition rate increases with the viscosity ratio, and the linear relationship between oil recovery factor and the square root of time is interestingly observed for $\lambda=5$ and $\lambda=10$, also like in the dripping regime. 

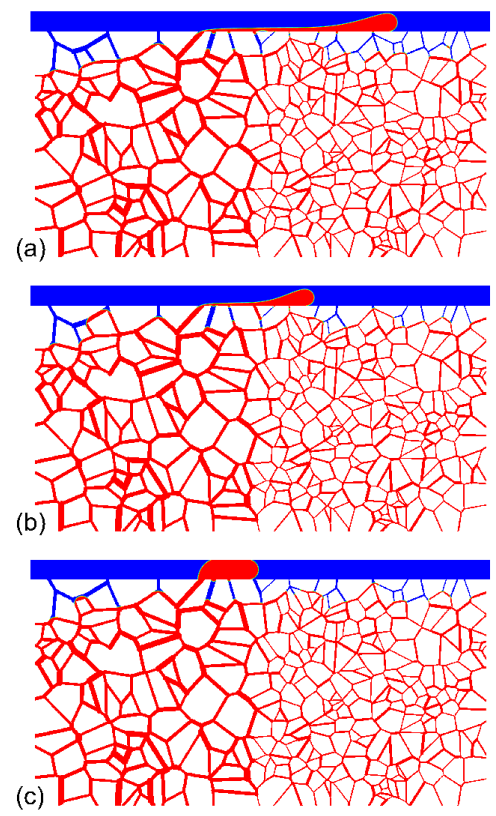
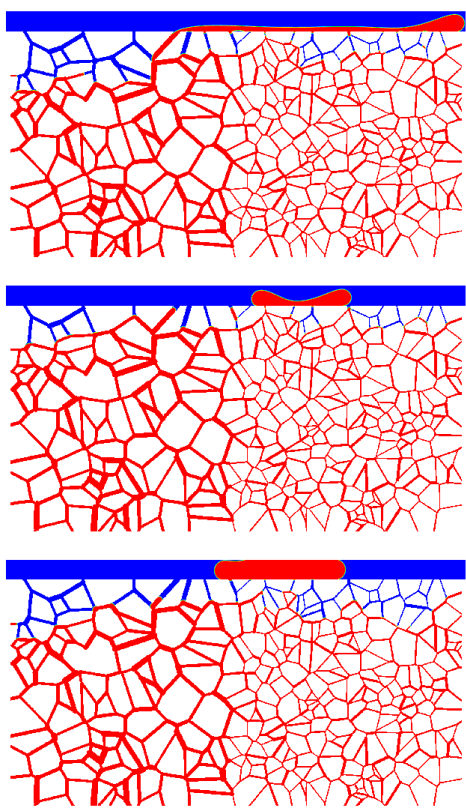
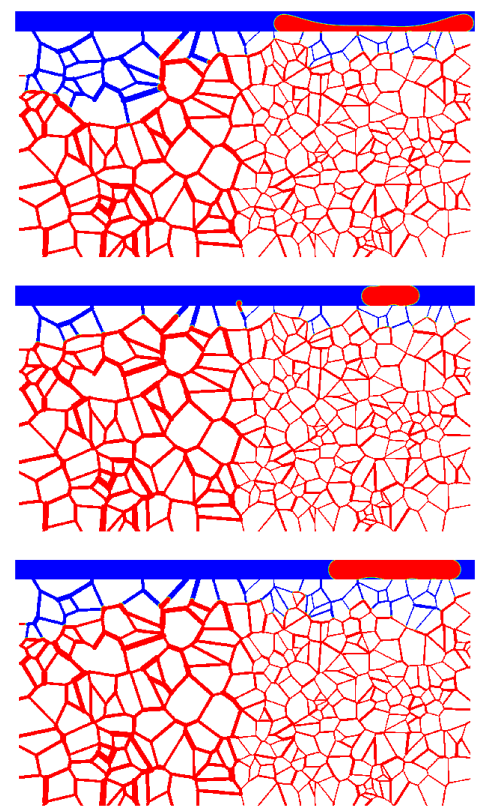

FIG. 15. Snapshots of the fluid distributions during imbibition for different viscosity ratios: (a) $\lambda=1$; (b) $\lambda=0.1$ and (c) $\lambda=10$. Note that the water and the oil are shown in blue and red respectively.

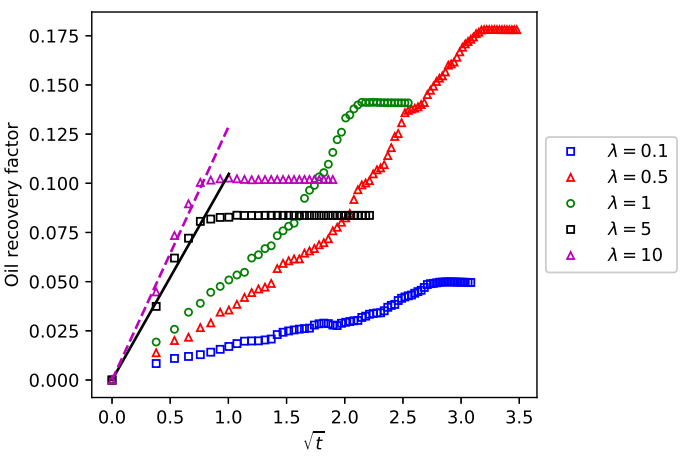

FIG. 16. Time evolution of the oil recovery factor for the viscosity ratios of $0.1,0.5,1,5$ and 10 . Note that the solid and dashed lines represent the linear fits to the data of $\lambda=5$ and $\lambda=10$, respectively, which are given by $r f=0.10439 \times t^{0.5}$ and $r f=0.12858 \times t^{0.5}$.

\section{E. Effect of fracture spacing}

In standard "water-flooding" oil recovery, the fracture spacing is an important characteristic of natural fractured rock ${ }^{62-65}$ that affects the water supply, the transport of expelled oil and thus the amount of recoverable oil. It is also important in artificially created fracture network during unconventional gas \& oil extraction to optimize the production rate and reduce the economic costs ${ }^{66-69}$. To investigate the effect of fracture spacing on the counter-current imbibition, we add another horizontal fracture in the primary geometry. By varying the spacing between the added fracture with the original one, we can ob- serve the effect of fracture spacing on the dynamic imbibition process and the ultimate oil recovery factor. Eight different fracture spacings are considered, where the fracture spacings normalized by the matrix height are $\delta=0.167,0.333,0.5$, $0.667,0.74,0.81,0.833$ and 0.88 , respectively.

Figure 17 shows the steady-state fluid distributions for different fracture spacings where the result of the primary geometry with a single fracture is also shown for comparison. From this figure, it can be seen that the interaction between the water fronts from different fractures is greatly affected by the fracture spacing. Specifically, when two fractures are very close to each other [Fig. 17(b)], the oil in the porous matrix located between two fractures is quickly expelled out, and thus only the lower fracture supplies water for further imbibition into the lower porous region. By increasing the fracture spacing, the water fronts from two fractures have more space to develop, and thus more oil is expelled into the fracture [Fig. 17(c) - (d)]. This explains why the oil recovery factor overall increases with the fracture spacing, which is shown in Fig. 18. However, when the fracture spacing $\delta$ is increased from 0.667 to 0.88 , the oil recovery factor does not increase but decrease instead (Fig. 18). This is because the lower fracture is too close to the matrix downside boundary, leading to dead throats in the lower porous region and thus restricting the development of water fronts, for example, at $\delta=0.833$ [see Fig. 17(f)].

\section{CONCLUSIONS}

A lattice Boltzmann color-gradient model is used for porescale simulation of the counter-current water-oil imbibition in 
(a)

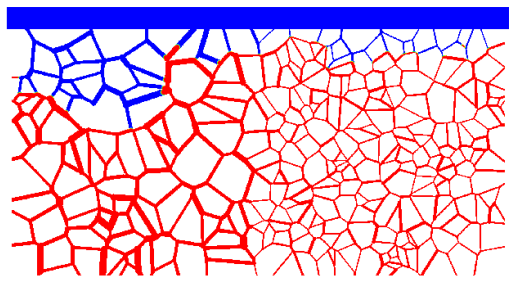

(d)

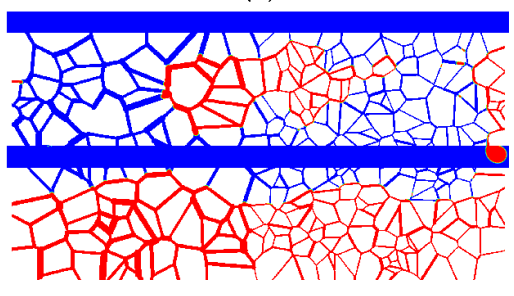

(b)

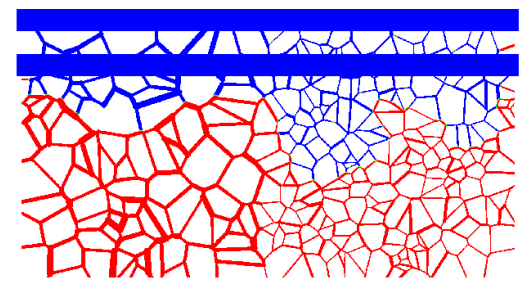

(e)

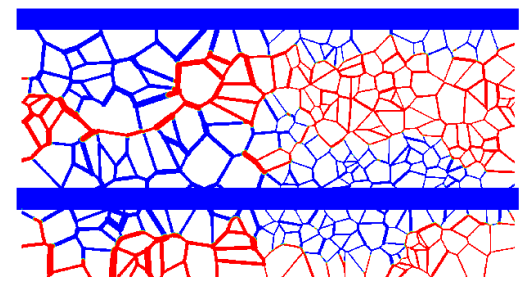

(c)

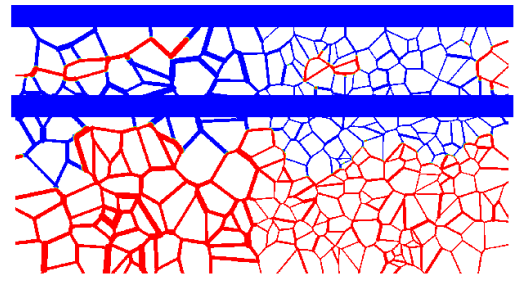

(f)

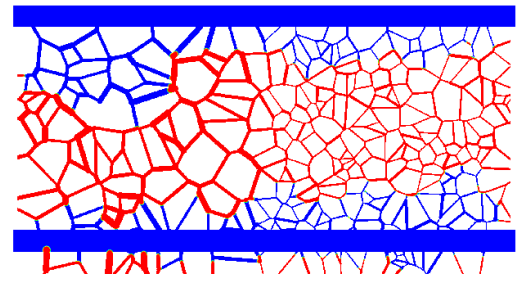

FIG. 17. Fluid distributions at steady-state for different fracture spacing: (a) $\delta=0$; (b) $\delta=0.167$; (c) $\delta=0.333$; (d) $\delta=0.5$; (e) $\delta=0.667$ and (f) $\delta=0.833$. Note that $\delta=0$ corresponds to the primary geometry with only one fracture. Note that the water and the oil are shown in blue and red respectively.

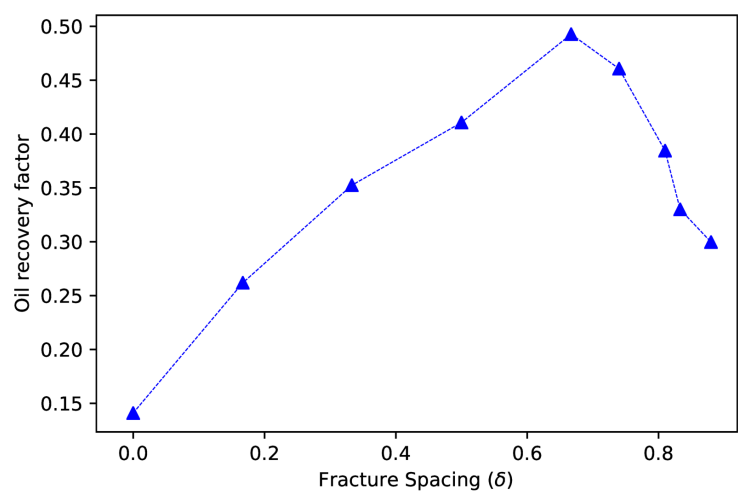

FIG. 18. Ultimate oil recovery factor as a function of the fracture spacing, which is normalized by the matrix height.

a strongly water-wet fracture-matrix system. The effects of water injection velocity into the fracture $\left(u_{\text {inj }}\right)$, geometry configuration of the high and low permeability zones, interfacial tension $(\sigma)$, viscosity ratio $(\lambda)$ of water to oil and the fracture spacing $(\delta)$ (if there are multiple fractures) on the dynamic imbibition process and the ultimate oil recovery factor are systematically analyzed. The main findings are summarized as follows.

The morphology of the expelled oil in the fracture changes with the water injection velocity and the interfacial tension. Depending on the relative strength of the viscous shear force and capillary force, the expelled oil in the fracture appears as either plugs or long threads or isolated drops. Accordingly, they are classified into three regimes, i.e. squeezing, jetting and dripping.

In the primary geometry, i.e. the one with left-wide porous matrix, we observe the counter-intuitive phenomenon that more water invades into the left-half region of the matrix (high permeability zone), which is due to that the oil plug entirely blocks the downstream fracture channel. The build-up pressure upstream facilitates water to invade the wider throats which improves the oil recovery factor. The ultimate oil recovery factor is not significantly affected by the injection velocity and is generally high in the squeezing regime; whereas in the jetting regime, it decreases gradually with the increase of injection velocity. In the dripping regime, the ultimate oil recovery factor is extremely low and we find that the amount of oil expelled out from the matrix scales with the square root of time, consistent with the previous experimental result ${ }^{56}$.

The geometry configuration of the high and low permeability zones affects the amount of oil that can be recovered by the counter-current imbibition in a fracture-matrix system. There exists a critical injection velocity above which water is preferably injected from the fracture end adjacent to the high permeability zone.

Decreasing interfacial tension favors reduction of the oil recovery as the oil morphology in the fracture shifts to the dripping regime. Increasing water-to-oil viscosity ratio leads to a higher water imbibition rate and the highest oil recovery factor is achieved at $\lambda=0.5$. Finally, introducing a second horizontal fracture is found to significantly enhance oil recovery. Increasing fracture spacing first leads to an increasing trend of the oil recovery factor, and then the highest oil recovery factor is reached when the fracture spacing is large enough to allow the water imbibition fronts from different fractures to develop independently. A further increase of the fracture spacing decreases the oil recovery factor as the lower fracture becomes too close to the matrix downside boundary. 


\section{ACKNOWLEDGMENTS}

This work is supported by the National Key Research and Development Project of China (No. 2016YFB0200902) and the National Natural Science Foundation of China (No.51876170). The authors would like to thank the "UK Consortium on Mesoscale Engineering Sciences (UKCOMES)" (http://www.ukcomes.org/) funded by the UK Engineering and Physical Sciences Research Council (EPSRC) under grant number EP/R029598/1 for providing computing time on ARCHER. And the simulations are also performed on ARCHIE-WeSt funded by EPSRC (EP/K000586/17). Qingqing $\mathrm{Gu}$ is grateful for the scholarship provided by China Scholarship Council and would like to thank Mr Hamidreza Erfani of University of Manchester for the beneficial discussions.

\section{Appendix A: Conversion between the lattice and physical units}

In the LB simulation, the physical quantities are represented in lattice units, for example, the time step $\delta_{t}$ is 1 , and the lattice spacing $\delta x$ is also 1 . Considering that the typical size of pores and throats in the natural porous media of interest is on the order of $10-100 \mu \mathrm{m}^{70}$, the following reference values are chosen to match the parameters in lattice units towards their physical values: the length scale $l_{0}=8 \times 10^{-6} \mathrm{~m}$, the mass scale $m_{0}=5.12 \times 10^{-13} \mathrm{~kg}$, and the time scale $t_{0}=7.2 \times 10^{-7} \mathrm{~s}$. Thus the physical length is obtained by $l^{p h y}=l_{0} l$, the density by $\rho^{p h y}=\rho m_{0} / l_{0}{ }^{3}$, and the interfacial tension by $\sigma^{p h y}=\sigma m_{0} / t_{0}^{2}$.

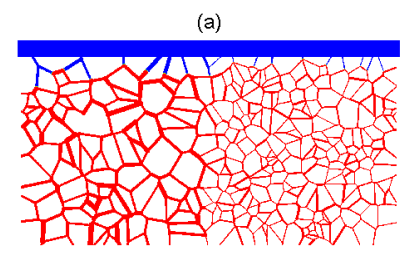

(c)

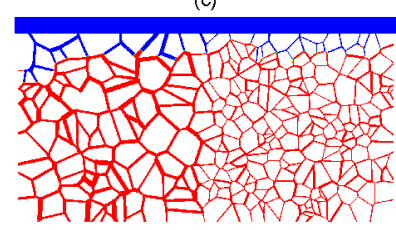

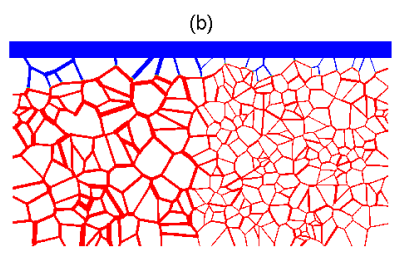

(d)

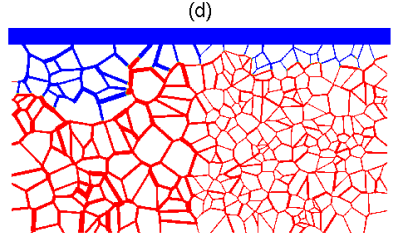

FIG. 19. Final fluid distributions for different contact angles: (a) $\theta=$ $\pi / 4$; (b) $\theta=\pi / 6$; (c) $\theta=\pi / 8$ and (d) $\theta=\pi / 10$. Note that the water and the oil are shown in blue and red, respectively.

\section{Appendix B: The effect of wettability}

To investigate the effect of wettability on the fluid displacement during the imbibition process, four preliminary tests have been carried out for the primary geometry with contact

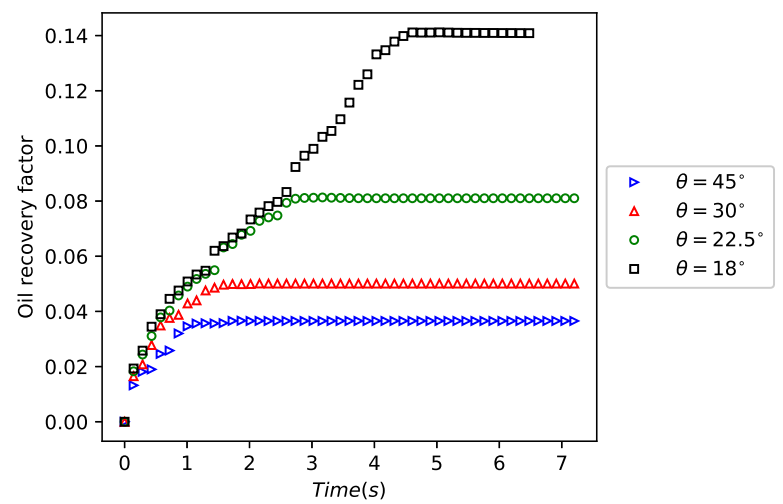

FIG. 20. The oil recovery factor as a function of time for the contact angles of $\pi / 4, \pi / 6, \pi / 8$ and $\pi / 10$.

angles of $\pi / 10, \pi / 8, \pi / 6$ and $\pi / 4$. The other parameters are chosen as the default values. Figure 19 shows the final fluid distributions for different contact angles. It can be observed that as the contact angle decreases from $\pi / 4$ to $\pi / 10$, the amount of water that can be imbibed into the matrix keeps increasing. Fig. 20 plots the time evolution of the oil recovery factor. It is seen that both the imbibition rate and the ultimate oil recovery increase as the contact angle decreases, consistent with the previous numerical results ${ }^{4}$.

\section{REFERENCES}

${ }^{1}$ A. M. Saidi, "Simulation of Naturally Fractured Reservoirs," in SPE Reservoir Simulation Symposium (1983) p. 12270.

${ }^{2}$ H. Li, H. Guo, Z. Yang, H. Ren, L. Meng, H. Lu, H. Xu, Y. Sun, T. Gao, and H. Zhang, "Evaluation of oil production potential in fractured porous media," Physics of Fluids 31, 052104 (2019).

${ }^{3}$ M. Pooladi-Darvish and A. Firoozabadi, "Cocurrent and Countercurrent Imbibition in a Water-Wet Matrix Block," SPE Journal 5, 3-11 (2000).

${ }^{4}$ M. R. Rokhforouz and H. A. Akhlaghi Amiri, "Phase-field simulation of counter-current spontaneous imbibition in a fractured heterogeneous porous medium," Physics of Fluids 29, 062104 (2017).

${ }^{5}$ C. C. Mattax and J. R. Kyte, "Imbibition Oil Recovery from Fractured, Water-Drive Reservoir," Society of Petroleum Engineers Journal 2, 177184 (1962).

${ }^{6}$ L. Cuiec, B. Bourbiaux, and F. Kalaydjian, "Oil Recovery by Imbibition in Low-Permeability Chalk," SPE Formation Evaluation 9, 200-208 (1994).

${ }^{7}$ X. Zhang, N. R. Morrow, and S. Ma, "Experimental Verification of a Modified Scaling Group for Spontaneous Imbibition," SPE Reservoir Engineering 11, 280-285 (1996).

${ }^{8}$ M. Shouxiang, N. R. Morrow, and X. Zhang, "Generalized scaling of spontaneous imbibition data for strongly water-wet systems," Journal of Petroleum Science and Engineering 18, 165-178 (1997).

${ }^{9}$ I. Jafari, M. Masihi, and M. Nasiri Zarandi, "Numerical simulation of counter-current spontaneous imbibition in water-wet fractured porous media: Influences of water injection velocity, fracture aperture, and grains geometry," Physics of Fluids 29, 113305 (2017).

${ }^{10} \mathrm{O}$. Torsaeter, "An Experimental Study of Water Imbibition in Chalk From the Ekofisk Field," in SPE Enhanced Oil Recovery Symposium (1984). 
${ }^{11}$ R. W. Parsons and P. R. Chaney, "Imbibition Model Studies on WaterWet Carbonate Rocks," Society of Petroleum Engineers Journal 6, 26-34 (1966).

${ }^{12} \mathrm{P}$. Blair, "Calculation of Oil Displacement by Countercurrent Water Imbibition," Society of Petroleum Engineers Journal 4, 195-202 (1964).

${ }^{13}$ A. M. Al Bahlani and T. Babadagli, "Heavy-Oil Recovery in Naturally Fractured Reservoirs with Varying Wettability by Steam Solvent CoInjection," in International Thermal Operations and Heavy Oil Symposium (2008).

${ }^{14}$ T. Babadagli, A. Al-Bemani, and F. Boukadi, "Analysis of Capillary Imbibition Recovery Considering the Simultaneous Effects of Gravity, Low IFT, and Boundary Conditions," Society of Petroleum Engineers, 57321 (1999).

${ }^{15}$ D. S. Schechter, D. Zhou, and F. M. Orr, "Low IFT drainage and imbibition," Journal of Petroleum Science and Engineering 11, 283-300 (1994).

${ }^{16}$ E. R. Brownscombe and A. B. Dyes, "Water-imbibition Displacement-A Possibility for the Spraberry," in Drilling and Production Practice (American Petroleum Institute, 1952) p. 383.

${ }^{17}$ R. Lenormand, C. Zarcone, and A. Sarr, "Mechanisms of the displacement of one fluid by another in a network of capillary ducts," Journal of Fluid Mechanics 135, 337-353 (1983).

${ }^{18}$ L. C. Chang, J. P. Tsai, H. Y. Shan, and H. H. Chen, "Experimental Study on Imbibition Displacement Mechanisms of Two-Phase Fluid Using Micro Model," Environmental Earth Sciences 59, 901-911 (2009).

${ }^{19}$ V. J. Niasar, S. M. Hassanizadeh, L. J. Pyrak-Nolte, and C. Berentsen, "Simulating drainage and imbibition experiments in a high-porosity micromodel using an unstructured pore network model," Water Resources Research 45, W02430 (2009).

${ }^{20}$ B. Yadali Jamaloei, K. Asghari, R. Kharrat, and F. Ahmadloo, "Pore-scale two-phase filtration in imbibition process through porous media at highand low-interfacial tension flow conditions," Journal of Petroleum Science and Engineering 72, 251-269 (2010).

${ }^{21}$ E. R. Rangel-German and A. R. Kovscek, "Experimental and analytical study of multidimensional imbibition in fractured porous media," Journal of Petroleum Science and Engineering 36, 45-60 (2002).

${ }^{22}$ I. Jafari, M. Masihi, and M. Nasiri Zarandi, "Experimental study on imbibition displacement mechanisms of two-phase fluid using micromodel: Fracture network, distribution of pore size, and matrix construction," Physics of Fluids 29, 122004 (2017).

${ }^{23}$ A. Gunde, T. Babadagli, S. S. Roy, and S. K. Mitra, "Pore-scale interfacial dynamics and oil-water relative permeabilities of capillary driven countercurrent flow in fractured porous media," Journal of Petroleum Science and Engineering 103, 106-114 (2013).

${ }^{24} \mathrm{Z}$. Xu, H. Liu, and A. J. Valocchi, "Lattice Boltzmann simulation of immiscible two-phase flow with capillary valve effect in porous media," Water Resources Research 53, 3770-3790 (2017).

${ }^{25}$ H. Huang, M. C. Sukop, and X.-Y. Lu, Multiphase Lattice Boltzmann Methods: Theory and Application (John Wiley \& Sons, 2015).

${ }^{26}$ H. Liu, Q. Kang, C. R. Leonardi, S. Schmieschek, A. Narváez, B. D. Jones, J. R. Williams, A. J. Valocchi, and J. Harting, "Multiphase Lattice Boltzmann Simulations for Porous Media Applications," Computational Geosciences , 1-51 (2015).

${ }^{27}$ Q. Gu, H. Liu, and Y. Zhang, "Lattice Boltzmann Simulation of Immiscible Two-Phase Displacement in Two-Dimensional Berea Sandstone," Applied Sciences 8, 1497 (2018).

${ }^{28}$ Tölke Jonas, Krafczyk Manfred, Schulz Manuel, and Rank Ernst, "Lattice Boltzmann simulations of binary fluid flow through porous media," Philosophical Transactions of the Royal Society of London. Series A: Mathematical, Physical and Engineering Sciences 360, 535-545 (2002).

${ }^{29}$ H. Huang, J.-J. Huang, and X.-Y. Lu, "Study of immiscible displacements in porous media using a color-gradient-based multiphase lattice Boltzmann method," Computers \& Fluids 93, 164-172 (2014).

${ }^{30}$ Y. Chen, Y. Li, A. J. Valocchi, and K. T. Christensen, "Lattice Boltzmann simulations of liquid $\mathrm{CO} 2$ displacing water in a 2D heterogeneous micromodel at reservoir pressure conditions," Journal of Contaminant Hydrology Advances in Experimental Techniques, Validation of Modelling Tools and Uncertainty in Predictions from Pore to Field Scale, 212, 14-27 (2018).

${ }^{31} \mathrm{M}$. Xu and H. Liu, "Prediction of immiscible two-phase flow properties in a two-dimensional Berea sandstone using the pore-scale lattice Boltzmann simulation,” The European Physical Journal E 41, 124 (2018).
${ }^{32}$ J. Bear, Dynamics of Fluids in Porous Media (Courier Corporation, 2013).

${ }^{33}$ C. Zhang, M. Oostrom, J. W. Grate, T. W. Wietsma, and M. G. Warner, "Liquid CO2 Displacement of Water in a Dual-Permeability Pore Network Micromodel," Environmental Science \& Technology 45, 7581-7588 (2011).

${ }^{34}$ S. Lishchuk, C. Care, and I. Halliday, "Lattice Boltzmann algorithm for surface tension with greatly reduced microcurrents," Physical Review E 67, 036701 (2003).

${ }^{35}$ I. Halliday, R. Law, C. Care, and A. Hollis, "Improved simulation of drop dynamics in a shear flow at low Reynolds and capillary number," Physical Review E 73, 056708 (2006).

${ }^{36}$ I. Halliday, A. Hollis, and C. Care, "Lattice Boltzmann algorithm for continuum multicomponent flow," Physical Review E 76, 026708 (2007).

${ }^{37}$ Y. Yu, H. Liu, Y. Zhang, and D. Liang, "Color-gradient lattice Boltzmann modeling of immiscible two-phase flows on partially wetting surfaces," Proceedings of the Institution of Mechanical Engineers, Part C: Journal of Mechanical Engineering Science 232, 416-430 (2018).

${ }^{38} \mathrm{Y}$. Zu and S. He, "Phase-field-based lattice Boltzmann model for incompressible binary fluid systems with density and viscosity contrasts," Physical Review E 87, 043301 (2013).

${ }^{39}$ I. Ginzburg, "Equilibrium-type and link-type lattice Boltzmann models for generic advection and anisotropic-dispersion equation," Advances in Water Resources 28, 1171-1195 (2005).

${ }^{40}$ I. Ginzburg, F. Verhaeghe, and D. d'Humieres, "Two-relaxation-time lattice Boltzmann scheme: About parametrization, velocity, pressure and mixed boundary conditions," Communications in Computational Physics 3, 427-478 (2008).

${ }^{41}$ I. Ginzburg, F. Verhaeghe, and D. d'Humieres, "Study of simple hydrodynamic solutions with the two-relaxation-times lattice Boltzmann scheme," Communications in Computational Physics 3, 519-581 (2008).

${ }^{42} \mathrm{Z}$. Yu and L. Fan, "Multirelaxation-time interaction-potential-based lattice Boltzmann model for two-phase flow," Physical Review E 82, 046708 (2010).

${ }^{43}$ L. Talon, D. Bauer, N. Gland, S. Youssef, H. Auradou, and I. Ginzburg, "Assessment of the two relaxation time Lattice-Boltzmann scheme to simulate Stokes flow in porous media," Water Resources Research 48, W04526 (2012).

${ }^{44}$ C. Pan, L.-S. Luo, and C. T. Miller, "An evaluation of lattice Boltzmann schemes for porous medium flow simulation," Computers \& Fluids Proceedings of the First International Conference for Mesoscopic Methods in Engineering and Science, 35, 898-909 (2006).

${ }^{45} \mathrm{D}$. d'Humières and I. Ginzburg, "Viscosity independent numerical errors for Lattice Boltzmann models: From recurrence equations to "magic" collision numbers," Computers \& Mathematics with Applications Mesoscopic Methods in Engineering and Science, 58, 823-840 (2009).

${ }^{46}$ P. Lallemand and L.-S. Luo, "Theory of the lattice Boltzmann method: Dispersion, dissipation, isotropy, Galilean invariance, and stability," Physical Review E 61, 6546 (2000).

${ }^{47}$ I. Ginzburg and D. d'Humieres, "Multireflection boundary conditions for lattice Boltzmann models," Physical Review E 68, 066614 (2003).

${ }^{48}$ J. U. Brackbill, D. B. Kothe, and C. Zemach, "A continuum method for modeling surface tension," Journal of Computational Physics 100, 335-354 (1992).

${ }^{49}$ Z. Guo, C. Zheng, and B. Shi, "Discrete lattice effects on the forcing term in the lattice Boltzmann method," Physical Review E 65, 046308 (2002).

${ }^{50}$ M. Latva-Kokko and D. H. Rothman, "Diffusion properties of gradientbased lattice Boltzmann models of immiscible fluids," Physical Review E 71, 056702 (2005).

${ }^{51}$ D. Debnath, J. S. Gainer, C. Kilic, D. Kim, K. T. Matchev, and Y. Yang, "Identifying phase-space boundaries with Voronoi tessellations," The European Physical Journal C 76, 645 (2016).

${ }^{52}$ M. J. Blunt, "Pore level modeling of the effects of wettability," SPE Journal 2, 494-510 (1997).

${ }^{53}$ A. J. C. Ladd, "Numerical simulations of particulate suspensions via a discretized Boltzmann equation. Part 1. Theoretical foundation," Journal of Fluid Mechanics 271, 285-309 (1994).

${ }^{54}$ C. U. Hatiboglu and T. Babadagli, "Pore-scale studies of spontaneous imbibition into oil-saturated porous media," Physical Review E 77, 066311 (2008). 
${ }^{55}$ N. R. Morrow and G. Mason, "Recovery of oil by spontaneous imbibition," Current Opinion in Colloid \& Interface Science 6, 321-337 (2001).

${ }^{56}$ E. R. Rangel-German and A. R. Kovscek, "A micromodel investigation of two-phase matrix-fracture transfer mechanisms," Water Resources Research 42, W03401 (2006).

${ }^{57}$ J. S. Rowlinson and B. Widom, Molecular Theory of Capillarity (Courier Corporation, 2013).

${ }^{58}$ P. Garstecki, M. J. Fuerstman, H. A. Stone, and G. M. Whitesides, "Formation of droplets and bubbles in a microfluidic T-junction-scaling and mechanism of break-up," Lab on a Chip 6, 437-446 (2006).

${ }^{59}$ M. D. Menech, P. Garstecki, F. Jousse, and H. A. Stone, "Transition from squeezing to dripping in a microfluidic T-shaped junction," Journal of Fluid Mechanics 595, 141-161 (2008).

${ }^{60}$ H. Liu and Y. Zhang, "Droplet formation in a T-shaped microfluidic junction,” Journal of Applied Physics 106, 034906 (2009).

${ }^{61}$ C. U. Hatiboglu and T. Babadagli, "Oil recovery by counter-current spontaneous imbibition: Effects of matrix shape factor, gravity, IFT, oil viscosity, wettability, and rock type,' Journal of Petroleum Science and Engineering 59, 106-122 (2007).

${ }^{62}$ D. T. Snow, "Rock Fracture Spacings, Openings, and Porosities,” Journal of the Soil Mechanics and Foundations Division 94, 73-92 (1968).

${ }^{63}$ T. Bai, D. D. Pollard, and H. Gao, "Explanation for fracture spacing in layered materials," Nature 403, 753-756 (2000).
${ }^{64}$ O. J. Ortega, R. A. Marrett, and S. E. Laubach, “A scale-independent approach to fracture intensity and average spacing measurement," AAPG Bulletin 90, 193-208 (2006).

${ }^{65} \mathrm{~J}$. Gong and W. R. Rossen, "Characteristic fracture spacing in primary and secondary recovery for naturally fractured reservoirs," Fuel 223, 470-485 (2018).

${ }^{66}$ N. P. Roussel and M. M. Sharma, "Optimizing Fracture Spacing and Sequencing in Horizontal-Well Fracturing," SPE Production \& Operations 26, 173-184 (2011).

${ }^{67}$ J. Morrill and J. L. Miskimins, "Optimization of Hydraulic Fracture Spacing in Unconventional Shales," in SPE Hydraulic Fracturing Technology Conference (Society of Petroleum Engineers, 2012).

${ }^{68} \mathrm{~K}$. Wu and J. E. Olson, "Investigation of the Impact of Fracture Spacing and Fluid Properties for Interfering Simultaneously or Sequentially Generated Hydraulic Fractures,' SPE Production \& Operations 28, 427-436 (2013).

${ }^{69}$ C. Jin, L. Sierra, and M. Mayerhofer, "A Production Optimization Approach to Completion and Fracture Spacing Optimization for Unconventional Shale Oil Exploitation," in Unconventional Resources Technology Conference, Denver, Colorado, 12-14 August 2013 (Society of Exploration Geophysicists, American Association of Petroleum Geologists, Society of Petroleum Engineers, Denver, Colorado, USA, 2013) pp. 601-612.

${ }^{70}$ M. Kim, A. Sell, and D. Sinton, “Aquifer-on-a-Chip: Understanding porescale salt precipitation dynamics during CO 2 sequestration," Lab on a Chip 13, 2508-2518 (2013). 